This is the peer reviewed version of the following article: “Nuño, E., Aldana, and Basañez, L. (2017) Task space consensus in networks of heterogeneous and uncertain robotic systems with variable time-delays, 31 (6): 917-937." which has been published in final form at [doi: 10.1002/acs.2738]. This article may be used for non-commercial purposes in accordance with Wiley Terms and Conditions for Self-Archiving." 


\title{
Task space consensus in networks of heterogeneous and uncertain robotic systems with variable time-delays
}

\author{
Emmanuel Nuño ${ }^{1, *, \dagger}$, Carlos I. Aldana ${ }^{1}$ and Luis Basañez ${ }^{2}$ \\ ${ }^{1}$ Department of Computer Science (DCC), University of Guadalajara (UdG). Guadalajara, Mexico \\ ${ }^{2}$ Institute of Industrial and Control Engineering (IOC), Technical University of Catalonia (UPC). Barcelona, Spain
}

\begin{abstract}
SUMMARY
This work deals with the leader-follower and the leaderless consensus problems in networks of multiple robot manipulators. The robots are non-identical, kinematically different (heterogeneous), and their physical parameters are uncertain. The main contribution of this work is a novel controller that solves the two consensus problems, in the task space, with the following features: it estimates the kinematic and the dynamic physical parameters; it is robust to interconnecting variable-time delays; it employs the singularity-free unitquaternions to represent the orientation; and, using energy-like functions, the controller synthesis follows a constructive procedure. Simulations using a network with four heterogeneous manipulators illustrate the performance of the proposed controller. Copyright (C) 2016 John Wiley \& Sons, Ltd.
\end{abstract}

Received 9 March 2015; Revised 14 September 2016; Accepted 19 September 2016

KEY WORDS: adaptive control; model reference adaptive control; time-delays; multi-agent systems

\section{INTRODUCTION}

The task space is a subspace of the Special Euclidean space of dimension three, denoted $S E(3)$. The pose of an object in the $S E(3)$ is composed of the position and the orientation (attitude). Task space control plays a major role in cooperative tasks performed by a network of multiple robot manipulators primarily if they are kinematically and dynamically dissimilar (heterogeneous) $[1,2]$. The control objectives in these systems are network consensus [3-5], synchronization [6-9], flocking $[10,11]$, and coordination $[12,13]$. The practical applications of multi-robot systems span different areas such as: underwater and space exploration (underwater cultural heritage recovery, coordination of clusters of satellites, and synchronization of spacecrafts) [14-16]; hazardous environments (search and rescue missions, military operations, and robot teleoperation) [17-20] and service robotics (commercial cleaning, material handling, furniture assembly, etc.) [21-23].

This paper focusses on finding the solution to the leader-follower and the leaderless consensus problems in networks of heterogeneous robot manipulators with uncertain physical parameters and interconnecting variable time-delays. In the leader-follower consensus, the objective is to ensure that all manipulators converge to a given leader pose, while in the leaderless consensus, the robot manipulators have to reach a certain agreement pose (consensus point). The solutions to these problems have been widely studied for first and second-order linear time invariant systems [24-28]. Solutions for different nonlinear Euler-Lagrange (EL) systems in the generalized coordinates (joint space) are reported in [9, 29-35], and in [36-39], for a more general class of nonlinear systems. $[1,6,30]$ present the solution to the the leader-follower case in the joint space, provided that the leader position is available to all the agents, and $[9,29,40]$ propose solutions to the

\footnotetext{
*Correspondence to: Emmanuel Nuño, Department of Computer Science (DCC), University of Guadalajara (UdG). Guadalajara, Mexico.

†E-mail: emmanuel.nuno@cucei.udg.mx
} 
leader-follower case in which the leader position is available only to a subset of the followers. [31] reports a solution to the leaderless case using simple proportional plus damping controllers. [32] proposes a proportional controller and a velocity estimator to solve the leaderless consensus problem. Recently, Liu et al. [41] have proposed a solution to both consensus problems without interconnecting delays, and [35] solves the leader-follower synchronization without interconnecting delays using an adaptive sliding controller. All these previous results deal with the consensus problems in the joint space.

Other interesting papers solve the consensus problems accounting only for the orientation (attitude) part. Among these are the following: [7] that provides the solution to both consensus problems for groups of rigid bodies in the presence of communication delays; [42] that provides three synchronization cases for the attitude alignment of spacecrafts; [43] where a passivity-based velocity input law is developed to achieve attitude synchronization; [44] that solves the attitude leaderless consensus without interconnection delays; and [45] that, using passivity-based controllers, solves a group orientation agreement in networks of rigid bodies provided the interconnection graph is undirected and without delays. However, for multiple degrees-of-freedom (DoF) mechanical systems, position, and orientation dynamics cannot be decoupled - because the inertia matrix is not, in general, block diagonal — and thus, the complete pose dynamics are needed for a formal stability analysis.

Some few exceptional works in the literature that deal with the complete pose consensus are the following: [1] that employ a minimal orientation representation for the leader-follower consensus; [46] that tackles the bilateral teleoperation of a network of multiple robots, a problem that can be seen as a special case of the leaderless consensus case; [47] that employs unit-quaternions and a PD plus damping and gravity compensation scheme for bilateral teleoperators with constant delays; [8] that solves the leaderless and leader-follower consensus problems using only a first order nonlinear kinematical model in the $S E(3)$; and [12], where a dual quaternion solution is provided for the coordination of the pose of rigid body networks. The last two works obviate the second order dynamic model, and they only deal with the kinematic model, without accounting for parameter uncertainty. Furthermore, in [2], it shown another solution to both problems without relying on velocity measurements; however, such work does not deal with parametric uncertainty, and it is not robust to interconnecting delays.

This work is closely related to two insightful papers [48, 49]. On one hand, [48] using similar kinematic and dynamic adaptation schemes as in [50, 51], solves the leader-follower consensus provided that the leader position is available to all the agents and, on the other hand, [49] solves the leaderless consensus problem without time-delays. Furthermore, for the orientation, these works make use of a minimal representation that exhibits singularities, and it is assumed that all the agents are kinematically similar, that is, all agents have the same DoF.

The main contribution of this work is a novel controller that solves the leader-follower and leaderless pose consensus problems for heterogeneous robot networks with uncertain kinematic and dynamic parameters. The main features of the proposed scheme are the following: it estimates the kinematic and the dynamic physical parameters; it is robust to interconnecting variable-time delays; it employs the singularity-free unit-quaternions to represent the orientation; and, using energy-like functions, the controller synthesis follows a constructive procedure. Moreover, in the leader-follower case, it is only assumed that at least one agent can access the leader's pose. Up to the authors' knowledge, this is the first work that solves these consensus problems with all these features. Furthermore, in order to show the performance of the proposed scheme, simulations using a network with four heterogeneous agents are also presented.

The following notation is used throughout the paper. $\mathbb{R}:=(-\infty, \infty), \mathbb{R}_{>0}:=(0, \infty), \mathbb{R}_{\geqslant 0}:=$ $[0, \infty) .|\mathbf{x}|$ stands for the standard Euclidean norm of vector $\mathbf{x}$. $\mathbf{I}_{k}$ and $\boldsymbol{\emptyset}_{k}$ represent the identity and all-zero matrices of size $k \times k$. Both $\mathbf{1}_{k}$ and $\mathbf{0}_{k}$ represent column vectors of size $k$ with all entries equal to one and to zero, respectively. The spectrum of the square matrix $\mathbf{A}$ is denoted by $\sigma(\mathbf{A})$ where the minimum and the maximum of its spectrum are denoted by $\sigma_{\min }(\mathbf{A})$ and $\sigma_{\max }(\mathbf{A})$, respectively. For any matrix $\mathbf{A} \in \mathbb{R}^{n \times m}, \mathbf{A}^{\top}\left(\mathbf{A A}^{\top}\right)^{-1}$ is its Moore-Penrose pseudo-inverse matrix denoted by $\mathbf{A}^{\dagger}$. For any function $\mathbf{f}: \mathbb{R}_{\geqslant 0} \rightarrow \mathbb{R}^{n}$, the $\mathcal{L}_{\infty}$-norm is defined as $\|\mathbf{f}\|_{\infty}:=\sup _{t \geqslant 0}|\mathbf{f}(t)|$, $\mathcal{L}_{2}$-norm as $\|\mathbf{f}\|_{2}:=\left(\int_{0}^{\infty}|\mathbf{f}(t)|^{2} d t\right)^{1 / 2}$. The $\mathcal{L}_{\infty}$ and $\mathcal{L}_{2}$ spaces are defined as the sets $\left\{\mathbf{f}: \mathbb{R}_{\geqslant 0} \rightarrow\right.$ 
$\left.\mathbb{R}^{n} \mid\|\mathbf{f}\|_{\infty}<\infty\right\}$ and $\left\{\mathbf{f}: \mathbb{R}_{\geqslant 0} \rightarrow \mathbb{R}^{n} \mid\|\mathbf{f}\|_{2}<\infty\right\}$, respectively. The argument of all time dependent signals is omitted, for example, $\mathbf{x} \equiv \mathbf{x}(t)$, except for those which are time-delayed, for example, $\mathbf{x}(t-T(t))$. The subscript $i \in \bar{N}:=\{1, \ldots, N\}$, where $N$ is the number of nodes of the network.

\section{SYSTEM DYNAMICS}

The dynamical behavior of the network accounts for a threefold: i) the dynamics of nodes, which are represented by EL-systems; ii) the interconnection topology, which is modeled using graph theory; and iii) the orientation description in the $S E(3)$, which is performed using unit-quaternions.

\subsection{Node dynamics}

The $i$ th-node is modeled as $n_{i}$-DoF robot manipulator ${ }^{\ddagger}$. Its EL-equation of motion, in joint space, is given by

$$
\mathbf{M}_{i}\left(\mathbf{q}_{i}\right) \ddot{\mathbf{q}}_{i}+\mathbf{C}_{i}\left(\mathbf{q}_{i}, \dot{\mathbf{q}}_{i}\right) \dot{\mathbf{q}}_{i}+\mathbf{g}_{i}\left(\mathbf{q}_{i}\right)=\boldsymbol{\tau}_{i}
$$

where $\mathbf{q}_{i}, \dot{\mathbf{q}}_{i}, \ddot{\mathbf{q}}_{i} \in \mathbb{R}^{n_{i}}$, are the joint positions, velocities, and accelerations, respectively; $\mathbf{M}_{i}\left(\mathbf{q}_{i}\right) \in$ $\mathbb{R}^{n_{i} \times n_{i}}$ is the symmetric and positive definite inertia matrix; $\mathbf{C}_{i}\left(\mathbf{q}_{i}, \dot{\mathbf{q}}_{i}\right) \in \mathbb{R}^{n_{i} \times n_{i}}$ is the Coriolis and centrifugal effects matrix, defined via the Christoffel symbols of the first kind; $\mathbf{g}_{i}\left(\mathbf{q}_{i}\right) \in \mathbb{R}^{n_{i}}$ is the gravitational torques vector and $\boldsymbol{\tau}_{i} \in \mathbb{R}^{n_{i}}$ is the torque exerted by the actuators (motors).

The pose of the $i$ th-end-effector, relative to a common reference frame, is denoted by the vector $\mathbf{x}_{i} \subset \mathbb{R}^{7}$, and it is composed of the position vector $\mathbf{p}_{i} \in \mathbb{R}^{3}$ and the orientation unit-quaternion ${ }^{\S}$ $\boldsymbol{\xi}_{i} \in S^{3}$, such that $\mathbf{x}_{i}:=\left[\mathbf{p}_{i}^{\top}, \boldsymbol{\xi}_{i}^{\top}\right]^{\top}$.

The kinematic relation between the joint velocities and the linear $\dot{\mathbf{p}}_{i}$ and angular $\boldsymbol{\omega}_{i}$ velocities of the $i$ th-end-effectors relative to a common reference frame, is given by

$$
\boldsymbol{v}_{i}=\left[\begin{array}{c}
\dot{\mathbf{p}}_{i} \\
\boldsymbol{\omega}_{i}
\end{array}\right]=\mathbf{J}_{i}\left(\mathbf{q}_{i}\right) \dot{\mathbf{q}}_{i}
$$

where $\boldsymbol{v}_{i} \in \mathbb{R}^{6}$ and $\mathbf{J}_{i}\left(\mathbf{q}_{i}\right) \in \mathbb{R}^{6 \times n_{i}}$ is the geometric Jacobian matrix.

It is assumed that each manipulator is an open serial chain with a certain combination of revolute and prismatic joints such that the inertia matrix is lower and upper bounded [52]. Under this assumption, the EL-system (1) enjoys the following properties [53-55]:

P1. For all $\mathbf{q}_{i} \in \mathbb{R}^{n_{i}}$, exist $\underline{M}_{i}, \bar{M}_{i} \in \mathbb{R}_{>0}$ such that $\underline{M}_{i} \leqslant\left\|\mathbf{M}_{i}\left(\mathbf{q}_{i}\right)\right\| \leqslant \bar{M}_{i}$.

P2. Matrix $\dot{\mathbf{M}}_{i}\left(\mathbf{q}_{i}\right)-2 \mathbf{C}_{i}\left(\mathbf{q}_{i}, \dot{\mathbf{q}}_{i}\right)$ is skew-symmetric.

P3. For any $\boldsymbol{\phi}_{i} \in \mathbb{R}^{n_{i}},(1)$ satisfies $\mathbf{M}_{i}\left(\mathbf{q}_{i}\right) \dot{\boldsymbol{\phi}}_{i}+\mathbf{C}_{i}\left(\mathbf{q}_{i}, \dot{\mathbf{q}}_{i}\right) \boldsymbol{\phi}_{i}-\mathbf{g}_{i}\left(\mathbf{q}_{i}\right)=\mathbf{Y}_{D i}\left(\mathbf{q}_{i}, \dot{\mathbf{q}}_{i}, \boldsymbol{\phi}_{i}, \dot{\boldsymbol{\phi}}_{i}\right) \boldsymbol{\theta}_{D i}$, where $\mathbf{Y}_{D i} \in \mathbb{R}^{n_{i} \times m_{D i}}$ is a regressor matrix of known functions, and $\boldsymbol{\theta}_{D i} \in \mathbb{R}^{m_{D i}}$ is a constant vector containing the dynamical parameters (link masses, moments of inertia, etc.).

P4. For all $\mathbf{q}_{i} \in \mathbb{R}^{n_{i}}$, the Jacobian matrix $\mathbf{J}_{i}\left(\mathbf{q}_{i}\right)$ is a bounded operator.

P5. The kinematic relation (2) satisfies $\boldsymbol{v}_{i}=\mathbf{Y}_{K i}\left(\mathbf{q}_{i}, \dot{\mathbf{q}}_{i}\right) \boldsymbol{\theta}_{K i}$, where $\mathbf{Y}_{K i} \in \mathbb{R}^{6 \times m_{K i}}$ is the kinematic regressor matrix, and $\boldsymbol{\theta}_{K i} \in \mathbb{R}^{m_{K i}}$ is a constant vector containing the kinematic physical parameters (link lengths, center of mass distances, etc.).

\subsection{Modeling the interconnection}

The interconnection of the $N$ followers is modeled using the Laplacian matrix $\mathbf{L}:=\left[\ell_{i j}\right] \in \mathbb{R}^{N \times N}$, whose elements are defined as

$$
\ell_{i j}=\left\{\begin{array}{cc}
\sum_{j \in \mathcal{N}_{i}} w_{i j} & i=j \\
-w_{i j} & i \neq j
\end{array}\right.
$$

\footnotetext{
${ }^{\ddagger}$ Note that each robot may have different number of DoF.

${ }^{\S}$ The set $S^{3} \subset \mathbb{R}^{4}$ represents an unitary sphere of dimension three and it is defined as $S^{3}:=\left\{\left.\xi \in \mathbb{R}^{4}|| \boldsymbol{\xi}\right|^{2}=1\right\}$.
} 
where $\mathcal{N}_{i}$ is the set of agents transmitting information to the $i$ th robot, $w_{i j}>0$ if $j \in \mathcal{N}_{i}$ and $w_{i j}=0$ otherwise.

Similar to passivity-based (energy-shaping) synchronization [4, 56-58] and in order to ensure that the interconnection forces are generated by the gradient of a potential function, the following assumption is used in this paper:

A1. The followers interconnection graph is undirected and connected.

\section{Remark 1}

Note that, by construction, $\mathbf{L}$ has a zero row sum, that is, $\mathbf{L} \mathbf{1}_{N}=\mathbf{0}_{N}$. Moreover, Assumption A1, ensures that $\mathbf{L}$ is symmetric, has a single zero-eigenvalue and the rest of the spectrum of $\mathbf{L}$ has positive real parts. Thus, $\operatorname{rank}(\mathbf{L})=N-1$.

In the leader-follower scenario, the Laplacian matrix models the followers interconnection and a diagonal matrix $\mathbf{A} \in \mathbb{R}^{N \times N}$ is used in this work to model the leader-follower interconnections. The following lemma, which is a special case of Lemma 3 of Hong et al. in [59] and Lemma 1.6 of Cao and Ren in [60], provides an interesting property of the composed Laplacian matrix $\mathbf{L}_{\ell}:=\mathbf{L}+\mathbf{A}$ that will be used in the proof of one of the main results. In fact, Lemma 1 provides a powerful tool for the controller design because, compared with other previous schemes - see [1, 30, 48, 61], to name a few- the leader-follower consensus problem (defined in Section 3.1) can be solved without assuming that the leader pose is available to all the followers.

\section{Lemma 1}

Consider a non-negative diagonal matrix $\mathbf{A}:=\operatorname{diag}\left(a_{1}, \ldots, a_{N}\right) \in \mathbb{R}^{N \times N}$ and suppose that, at least, one $a_{i}$ is strictly positive, that is, there exists some $a_{i}>0$. Assume that $\mathbf{A 1}$ holds, then the matrix $\mathbf{L}_{\ell}:=\mathbf{L}+\mathbf{A}$ is symmetric, positive definite and of full rank.

With regards to the interconnection time-delays, similar to other passivity-based schemes [62], it is assumed that:

A2. The information exchange, from the $j$-th agent to the $i$-th agent, is subject to a variable timedelay $T_{j i}(t)$ with a known upper-bound ${ }^{*} T_{j i}$. Hence, it holds that $0 \leqslant T_{j i}(t) \leqslant{ }^{*} T_{j i}<\infty$. Moreover, $\dot{T}_{j i}(t)$ is bounded.

For practical applications, if the time-delays are not known, then the first time-derivative of the delays can be computed using the following method. Along with the pose and the velocities, the value of a function $f(t)$ is sent through the communications. And then, when $f(t)$ arrives to its destination, it has the value $f(t-T(t))$. With this data, $\dot{T}(t)$ can be computed at both ends, indirectly, from the fact that $\dot{f}(t-T(t))=[1-\dot{T}(t)] \dot{f}(t)$. The way to do it is to design $f(t)$ such that $\dot{f}(t)=1$. In such a case, $\dot{T}(t)=1-\dot{f}(t-T(t))$. Finally, $\dot{f}(t-T(t))$ is calculated numerically and hence, $\dot{T}(t)$ can be obtained without knowledge of $T(t)$.

\subsection{On representing the orientation}

Compared with other orientation representations, for example, yaw-pitch-roll parameters, Euler angles, etc., the unit-quaternions are known to be free of singularities [63]. A unit-quaternion $\xi_{i} \in S^{3}$ can be split in two elements: one scalar term $\eta_{i} \in \mathbb{R}$ and one vectorial term $\boldsymbol{\beta}_{i} \in \mathbb{R}^{3}$. Thus, $\boldsymbol{\xi}_{i}:=\left[\eta_{i}, \boldsymbol{\beta}_{i}^{\top}\right]^{\top}$ and, from the unit norm constraint, $\eta_{i}^{2}+\boldsymbol{\beta}_{i}^{\top} \boldsymbol{\beta}_{i}=1$ (refer to [64, 65] for a detailed list of properties and operations involving unit-quaternions). The unit-quaternion $\boldsymbol{\xi}_{i}$ can be easily obtained from the rotation matrix $\mathbf{R}_{i} \in S O(3):=\left\{\mathbf{R}_{i} \in \mathbb{R}^{3 \times 3} \mid \mathbf{R}_{i}^{\top} \mathbf{R}_{i}=\mathbf{I}_{3}\right.$, $\left.\operatorname{det}\left(\mathbf{R}_{i}\right)=1\right\}$ [54]. Similarly, the Rodriguez formula [45] allows to obtain a rotation matrix corresponding to a given quaternion as 


$$
\mathbf{R}_{i}\left(\boldsymbol{\xi}_{i}\right)=\left(\eta_{i}^{2}-\boldsymbol{\beta}_{i}^{\top} \boldsymbol{\beta}_{i}\right) \mathbf{I}_{3}+2 \boldsymbol{\beta}_{i} \boldsymbol{\beta}_{i}^{\top}-2 \eta_{i} \mathbf{S}\left(\boldsymbol{\beta}_{i}\right)
$$

where $\mathbf{S}(\cdot)$ is the skew-symmetric matrix operator ${ }^{\mathrm{It}}$.

The orientation error, relative to the world frame, between two different frames, $\boldsymbol{\Sigma}_{i}$ and $\boldsymbol{\Sigma}_{j}$, can be described by the rotation matrix $\tilde{\mathbf{R}}_{i j}:=\mathbf{R}_{i} \mathbf{R}_{j}^{\top} \in S O(3)$. The unit-quaternion describing such orientation error is given by

$$
\begin{aligned}
\tilde{\boldsymbol{\xi}}_{i j}=\boldsymbol{\xi}_{i} \odot \boldsymbol{\xi}_{j}^{*}=\left[\begin{array}{c}
\tilde{\eta}_{i j} \\
\tilde{\boldsymbol{\beta}}_{i j}
\end{array}\right] & =\left[\begin{array}{c}
\boldsymbol{\xi}_{i}^{\top} \boldsymbol{\beta}_{j} \\
\eta_{j} \boldsymbol{\beta}_{i}-\eta_{i} \boldsymbol{\beta}_{j}-\mathbf{S}\left(\boldsymbol{\beta}_{i}\right) \boldsymbol{\beta}_{j}
\end{array}\right] \\
& =\left[\begin{array}{c}
\eta_{i} \eta_{j}+\boldsymbol{\beta}_{i}^{\top} \boldsymbol{\beta}_{j} \\
-\mathbf{U}^{\top}\left(\boldsymbol{\xi}_{i}\right) \boldsymbol{\xi}_{j}
\end{array}\right]
\end{aligned}
$$

where $\odot$ denotes the quaternion product, $\boldsymbol{\xi}_{(\cdot)}^{*}=\left[\eta_{(\cdot)},-\boldsymbol{\beta}_{(\cdot)}^{\top}\right]^{\top}$ is the quaternion conjugate of $\boldsymbol{\xi}_{(\cdot)}$ and $\mathbf{U}\left(\xi_{i}\right)$ is defined as

$$
\mathbf{U}\left(\boldsymbol{\xi}_{i}\right):=\left[\begin{array}{c}
-\boldsymbol{\beta}_{i}^{\top} \\
\eta_{i} \mathbf{I}_{3}-\mathbf{S}\left(\boldsymbol{\beta}_{i}\right)
\end{array}\right]
$$

The normality condition and some straightforward calculations show that $\tilde{\boldsymbol{\beta}}_{i j}=\mathbf{0}$ if and only if $\xi_{i}= \pm \xi_{j}$. This, in turn, implies that $\mathbf{U}^{\top}\left(\boldsymbol{\xi}_{i}\right) \boldsymbol{\xi}_{j}=\mathbf{0}_{3}$, this last due to the fact that

$$
\mathbf{U}^{\top}\left(\boldsymbol{\xi}_{i}\right) \boldsymbol{\xi}_{i}=\left[\begin{array}{ll}
-\boldsymbol{\beta}_{i} & \eta_{i} \mathbf{I}_{3}+\mathbf{S}\left(\boldsymbol{\beta}_{i}\right)
\end{array}\right]\left[\begin{array}{c}
\eta_{i} \\
\boldsymbol{\beta}_{i}
\end{array}\right]=\mathbf{0}_{3} .
$$

where the property $\mathbf{S}(\mathbf{a}) \mathbf{a}=\mathbf{0}_{3}$ is used. A key observation is that $\boldsymbol{\xi}_{i}=\boldsymbol{\xi}_{j}$ and $\boldsymbol{\xi}_{i}=-\boldsymbol{\xi}_{j}$ represent the same physical orientation [7, 45]. In fact, using (4), it can be easily established that $\mathbf{R}_{i}\left(\boldsymbol{\xi}_{i}\right)=$ $\mathbf{R}_{j}\left(\xi_{j}\right)$ if and only if $\boldsymbol{\xi}_{i}= \pm \boldsymbol{\xi}_{j}$.

The relation between the time-derivative of a unit-quaternion and the angular velocity, relative to the world reference frame, is given by

$$
\dot{\xi}_{i}=\frac{1}{2} \mathbf{U}\left(\xi_{i}\right) \omega_{i}
$$

Hence, defining $\boldsymbol{\Phi}\left(\boldsymbol{\xi}_{i}\right):=\operatorname{diag}\left(\mathbf{I}_{3}, \frac{1}{2} \mathbf{U}\left(\boldsymbol{\xi}_{i}\right)\right)$, it holds that

$$
\dot{\mathbf{x}}_{i}=\Phi\left(\xi_{i}\right) v_{i}
$$

The following properties have been borrowed from $[12,66-68]$ and are used throughout the rest of the paper.

P6. For all $\boldsymbol{\xi}_{i} \in S^{3}, \mathbf{U}^{\top}\left(\boldsymbol{\xi}_{i}\right) \mathbf{U}\left(\boldsymbol{\xi}_{i}\right)=\mathbf{I}_{3}$. Hence, $\operatorname{rank}\left(\mathbf{U}\left(\boldsymbol{\xi}_{i}\right)\right)=3$ and $\operatorname{ker}\left(\mathbf{U}^{\top}\left(\boldsymbol{\xi}_{i}\right)\right)=\operatorname{span}\left(\boldsymbol{\xi}_{i}\right)$.

P7. For all $\boldsymbol{\xi}_{i} \in S^{3}$ and $\dot{\boldsymbol{\xi}}_{i} \in \mathbb{R}^{4}, \dot{\mathbf{U}}\left(\boldsymbol{\xi}_{i}\right)=\mathbf{U}\left(\dot{\boldsymbol{\xi}}_{i}\right)$.

P8. Because, for all $\boldsymbol{\xi}_{i} \in S^{3},\left|\xi_{i}\right|=1$ then $\mathbf{U}\left(\xi_{i}\right)$ is a bounded operator.

Note that $\mathbf{P 6}$ implies, from (8), that $\left|\boldsymbol{\omega}_{i}\right|^{2}=4\left|\dot{\xi}_{i}\right|^{2}$.

\section{CONSENSUS IN THE TASK SPACE}

This section presents the formal statement of the leader-follower and the leaderless consensus problems together with our main results, which are the solutions to both problems.

$\overline{\mathbb{T}_{F}}$ For any $\mathbf{a}, \mathbf{b} \in \mathbb{R}^{3}, \mathbf{S}(\mathbf{a}) \mathbf{b}=\mathbf{a} \times \mathbf{b}$. Some well known properties of the skew-symmetric matrix operator, $\mathbf{S}(\cdot)$, used throughout the paper, are $\mathbf{S}(\mathbf{a})^{\top}=\mathbf{S}(-\mathbf{a})=-\mathbf{S}(\mathbf{a})$ and $\mathbf{S}(\mathbf{a}) \mathbf{a}=\mathbf{0}_{3}$. 


\subsection{Problem setting}

Consider a network of $N$ heterogeneous EL-systems of the form (1). Assume that the interconnection graph fulfills Assumptions A1 and A2. Furthermore, suppose that the kinematic and the dynamic physical parameters are uncertain. In this scenario, find the controllers to solve the following two consensus problems:

(a.) Leader-Follower Consensus Problem (LFCP): The network of $N$ followers has to be regulated at a given constant leader pose $\mathbf{x}_{\ell}:=\left[\mathbf{p}_{\ell}^{\top}, \boldsymbol{\xi}_{\ell}^{\top}\right]^{\top} \subset \mathbb{R}^{7}$, provided that the leader pose is only available to a certain nonempty set of followers. Hence, for all $i \in \bar{N}$,

$$
\lim _{t \rightarrow \infty}\left|\boldsymbol{v}_{i}(t)\right|=0, \quad \lim _{t \rightarrow \infty} \mathbf{x}_{i}(t)=\mathbf{x}_{\ell}
$$

(b.) Leaderless Consensus Problem (LCP): In the absence of a leader, the network of $N$ followers has to asymptotically reach a consensus pose, denoted $\mathbf{x}_{c}:=\left[\mathbf{p}_{c}^{\top}, \boldsymbol{\xi}_{c}^{\top}\right]^{\top} \subset \mathbb{R}^{7}$. Hence, for all $i \in \bar{N}$

$$
\lim _{t \rightarrow \infty}\left|\boldsymbol{v}_{i}(t)\right|=0, \quad \lim _{t \rightarrow \infty} \mathbf{x}_{i}(t)=\mathbf{x}_{c}
$$

\subsection{Leader-follower consensus problem}

As mentioned in the problem statement, this paper makes the following assumption for the leaderfollower interconnection:

A3. At least one of the $N$ follower robots has direct access to the leader's pose $\mathbf{x}_{\ell}$, that is, in the graph of $N+1$ nodes, being node zero the leader node, there exists at least one directed edge from the leader to any of the $N$ followers.

Assumptions A1 and A3 ensure that the leader pose is globally reachable from any of the $N$ follower nodes, that is, there exists a path from the leader to any follower robot.

Considering that the kinematic and the dynamics physical parameters are uncertain, and using (2), together with $\mathbf{P 3}$ and $\mathbf{P 5}$, it holds that

$$
\hat{\mathbf{J}}_{i}\left(\mathbf{q}_{i}\right) \dot{\mathbf{q}}_{i}=\mathbf{Y}_{K i}\left(\mathbf{q}_{i}, \dot{\mathbf{q}}_{i}\right) \hat{\boldsymbol{\theta}}_{K i}
$$

and $\hat{\mathbf{M}}_{i}\left(\mathbf{q}_{i}\right) \dot{\boldsymbol{\phi}}_{i}+\hat{\mathbf{C}}_{i}\left(\mathbf{q}_{i}, \dot{\mathbf{q}}_{i}\right) \boldsymbol{\phi}_{i}-\hat{\mathbf{g}}_{i}\left(\mathbf{q}_{i}\right)=\mathbf{Y}_{D i}\left(\mathbf{q}_{i}, \dot{\mathbf{q}}_{i}, \boldsymbol{\phi}_{i}, \dot{\boldsymbol{\phi}}_{i}\right) \hat{\boldsymbol{\theta}}_{D i}$, where $\boldsymbol{\phi}_{i} \in \mathbb{R}^{n_{i}}$ is a differentiable signal that will be defined later, $\hat{\boldsymbol{\theta}}_{K i} \in \mathbb{R}^{m_{K i}}$ and $\hat{\boldsymbol{\theta}}_{D i} \in \mathbb{R}^{m_{D i}}$ are the kinematic and the dynamics estimated physical parameters, respectively. $\hat{\mathbf{J}}_{i}\left(\mathbf{q}_{i}\right), \hat{\mathbf{M}}_{i}\left(\mathbf{q}_{i}\right), \hat{\mathbf{C}}_{i}\left(\mathbf{q}_{i}, \dot{\mathbf{q}}_{i}\right)$ are the estimated Jacobian, inertia and Coriolis matrices and $\hat{\mathbf{g}}_{i}\left(\mathbf{q}_{i}\right)$ is the estimated gravity vector.

Setting-up the controller

$$
\boldsymbol{\tau}_{i}=-\mathbf{Y}_{D i}\left(\mathbf{q}_{i}, \dot{\mathbf{q}}_{i}, \boldsymbol{\phi}_{i}, \dot{\boldsymbol{\phi}}_{i}\right) \hat{\boldsymbol{\theta}}_{D i}-K_{i} \hat{\mathbf{J}}_{i}^{\top}\left(\mathbf{q}_{i}\right) \hat{\mathbf{J}}_{i}\left(\mathbf{q}_{i}\right) \boldsymbol{\epsilon}_{i},
$$

where $K_{i} \in \mathbb{R}_{>0}$ and

$$
\boldsymbol{\epsilon}_{i}:=\dot{\mathbf{q}}_{i}+\phi_{i}
$$

yields the following closed-loop system

$$
\mathbf{M}_{i}\left(\mathbf{q}_{i}\right) \dot{\boldsymbol{\epsilon}}_{i}+\mathbf{C}_{i}\left(\mathbf{q}_{i}, \dot{\mathbf{q}}_{i}\right) \boldsymbol{\epsilon}_{i}+K_{i} \hat{\mathbf{J}}_{i}^{\top}\left(\mathbf{q}_{i}\right) \hat{\mathbf{J}}_{i}\left(\mathbf{q}_{i}\right) \boldsymbol{\epsilon}_{i}=\mathbf{Y}_{D i} \tilde{\boldsymbol{\theta}}_{D i},
$$

with $\tilde{\boldsymbol{\theta}}_{D i}:=\boldsymbol{\theta}_{D i}-\hat{\boldsymbol{\theta}}_{D i}$.

As usual in the adaptive control design, consider the following energy-like function

$$
\mathcal{V}_{i}=\frac{1}{2}\left[\boldsymbol{\epsilon}_{i}^{\top} \mathbf{M}_{i}\left(\mathbf{q}_{i}\right) \boldsymbol{\epsilon}_{i}+\tilde{\boldsymbol{\theta}}_{D i}^{\top} \boldsymbol{\Gamma}_{D i}^{-1} \tilde{\boldsymbol{\theta}}_{D i}\right]
$$

where $\boldsymbol{\Gamma}_{D i}=\boldsymbol{\Gamma}_{D i}^{\top}>0$. Evaluating $\dot{\mathcal{V}}_{i}$ along (15), using $\mathbf{P 2}$, the fact that $\dot{\tilde{\boldsymbol{\theta}}}_{D i}=-\dot{\hat{\boldsymbol{\theta}}}_{D i}$ and defining the dynamic parameters adaptation law as 


$$
\dot{\hat{\boldsymbol{\theta}}}_{D i}:=\boldsymbol{\Gamma}_{D i} \mathbf{Y}_{D i}^{\top} \boldsymbol{\epsilon}_{i}
$$

yields

$$
\dot{\mathcal{V}}_{i}=-K_{i}\left|\hat{\mathbf{J}}_{i}\left(\mathbf{q}_{i}\right) \boldsymbol{\epsilon}_{i}\right|^{2} \leqslant 0 .
$$

Interestingly but not surprising, because $\mathcal{V}_{i}$ is positive definite and radially unbounded with regards to $\boldsymbol{\epsilon}_{i}$ and $\tilde{\boldsymbol{\theta}}_{D i}$ and $\dot{\mathcal{V}}_{i} \leqslant 0$, the signals $\hat{\mathbf{J}}_{i}\left(\mathbf{q}_{i}\right) \boldsymbol{\epsilon}_{i} \in \mathcal{L}_{2}$ and $\boldsymbol{\epsilon}_{i}, \tilde{\boldsymbol{\theta}}_{D i} \in \mathcal{L}_{\infty}$ for any choice on the differentiable function $\phi_{i}$.

Now, pre-multiplying (14) by $\hat{\mathbf{J}}_{i}\left(\mathbf{q}_{i}\right)$ and using (2) and (12) yields $\hat{\mathbf{J}}_{i}\left(\mathbf{q}_{i}\right) \boldsymbol{\epsilon}_{i}=\boldsymbol{v}_{i}+\hat{\mathbf{J}}_{i}\left(\mathbf{q}_{i}\right) \boldsymbol{\phi}_{i}+$ $\mathbf{Y}_{K i}\left(\mathbf{q}_{i}, \dot{\mathbf{q}}_{i}\right) \tilde{\boldsymbol{\theta}}_{K i}$, where $\tilde{\boldsymbol{\theta}}_{K i}=\hat{\boldsymbol{\theta}}_{K i}-\boldsymbol{\theta}_{K i}$. Hence, $\dot{\mathcal{V}}_{i}$ can be rewritten as

$$
\dot{\mathcal{V}}_{i}=-K_{i}\left[\left|\boldsymbol{v}_{i}+\hat{\mathbf{J}}_{i}\left(\mathbf{q}_{i}\right) \boldsymbol{\phi}_{i}\right|^{2}+\left|\mathbf{Y}_{K i}\left(\mathbf{q}_{i}, \dot{\mathbf{q}}_{i}\right) \tilde{\boldsymbol{\theta}}_{K i}\right|^{2}\right]-2 K_{i} \tilde{\boldsymbol{\theta}}_{K i}^{\top} \mathbf{Y}_{K i}^{\top}\left(\mathbf{q}_{i}, \dot{\mathbf{q}}_{i}\right)\left[\boldsymbol{v}_{i}+\hat{\mathbf{J}}_{i}\left(\mathbf{q}_{i}\right) \boldsymbol{\phi}_{i}\right] .
$$

The form of $\dot{\mathcal{V}}_{i}$ motivates to propose the following function

$$
\mathcal{W}_{i}=\frac{1}{K_{i}} \mathcal{V}_{i}+\tilde{\boldsymbol{\theta}}_{K i}^{\top} \boldsymbol{\Gamma}_{K i}^{-1} \tilde{\boldsymbol{\theta}}_{K i},
$$

where $\Gamma_{K i}=\Gamma_{K i}^{\top}>0$. Thus, setting the kinematic parameters adaptation law as

$$
\dot{\hat{\boldsymbol{\theta}}}_{K i}:=\boldsymbol{\Gamma}_{K i} \mathbf{Y}_{K i}^{\top}\left(\mathbf{q}_{i}, \dot{\mathbf{q}}_{i}\right)\left[\boldsymbol{v}_{i}+\hat{\mathbf{J}}_{i}\left(\mathbf{q}_{i}\right) \boldsymbol{\phi}_{i}\right],
$$

ensures that

$$
\dot{\mathcal{W}}_{i}=-\left|\boldsymbol{v}_{i}\right|^{2}-2 \boldsymbol{v}_{i}^{\top} \hat{\mathbf{J}}_{i}\left(\mathbf{q}_{i}\right) \boldsymbol{\phi}_{i}-\left|\hat{\mathbf{J}}_{i}\left(\mathbf{q}_{i}\right) \boldsymbol{\phi}_{i}\right|^{2}-\left|\mathbf{Y}_{K i}\left(\mathbf{q}_{i}, \dot{\mathbf{q}}_{i}\right) \tilde{\boldsymbol{\theta}}_{K i}\right|^{2} .
$$

Because $\boldsymbol{v}_{i}$ is the linear and angular velocities vector, the crossed term $\boldsymbol{v}_{i}^{\top} \hat{\mathbf{J}}_{i}\left(\mathbf{q}_{i}\right) \boldsymbol{\phi}_{i}$ in the last equation, suggests the design of $\boldsymbol{\phi}_{i}$ as a function of the pose error. Hence, let us define

$$
\boldsymbol{\phi}_{i}:=\hat{\mathbf{J}}_{i}^{\dagger}\left(\mathbf{q}_{i}\right) \boldsymbol{\Phi}^{\top}\left(\boldsymbol{\xi}_{i}\right) \mathbf{e}_{i}
$$

where $\mathbf{e}_{i}$ is the pose error, between each manipulator with its leader and its neighbors, given by

$$
\mathbf{e}_{i}:=a_{i}\left(\mathbf{x}_{i}-\mathbf{x}_{\ell}\right)+\sum_{j \in \mathcal{N}_{i}} w_{i j}\left[\mathbf{x}_{i}-\mathbf{x}_{j}\left(t-T_{j i}(t)\right)\right],
$$

where $a_{i}>0$ if the leader's pose $\mathbf{x}_{\ell}$ is available to the $i$ th manipulator and $a_{i}=0$, otherwise. $\hat{\mathbf{J}}_{i}^{\dagger}$ is the Moore-Penrose pseudo-inverse of $\hat{\mathbf{J}}_{i}$ and it is given by $\hat{\mathbf{J}}_{i}^{\dagger}:=\hat{\mathbf{J}}_{i}^{\top}\left(\hat{\mathbf{J}}_{i} \hat{\mathbf{J}}_{i}^{\top}\right)^{-1}$.

Such definition of $\boldsymbol{\phi}_{i}$, together with (9), ensures that

$$
\dot{\mathcal{W}}_{i}=-\left|\boldsymbol{v}_{i}\right|^{2}-\left|\boldsymbol{\Phi}^{\top}\left(\boldsymbol{\xi}_{i}\right) \mathbf{e}_{i}\right|^{2}-\left|\mathbf{Y}_{K i} \tilde{\boldsymbol{\theta}}_{K i}\right|^{2}-2 \dot{\mathbf{x}}_{i}^{\top} \mathbf{e}_{i}
$$

Let us propose the total energy-like function $\mathcal{H}_{i}$ as

$$
\mathcal{H}_{i}=\mathcal{W}_{i}+a_{i}\left|\mathbf{x}_{i}-\mathbf{x}_{\ell}\right|^{2}+\frac{1}{2} \sum_{j \in \mathcal{N}_{i}} w_{i j}\left|\mathbf{x}_{i}-\mathbf{x}_{j}\right|^{2} .
$$

Using (22) and (23), $\dot{\mathcal{H}}_{i}$ is given by

$$
\dot{\mathcal{H}}_{i}=-\left|\boldsymbol{v}_{i}\right|^{2}-\left|\boldsymbol{\Phi}^{\top}\left(\boldsymbol{\xi}_{i}\right) \mathbf{e}_{i}\right|^{2}-\left|\mathbf{Y}_{K i} \tilde{\boldsymbol{\theta}}_{K i}\right|^{2}-2 \dot{\mathbf{x}}_{i}^{\top}\left(\mathbf{e}_{i}-a_{i}\left(\mathbf{x}_{i}-\mathbf{x}_{\ell}\right)\right)+\sum_{j \in \mathcal{N}_{i}} w_{i j}\left(\mathbf{x}_{i}-\mathbf{x}_{j}\right)^{\top}\left(\dot{\mathbf{x}}_{i}-\dot{\mathbf{x}}_{j}\right)
$$

and using the error definition (22) results

$$
\begin{aligned}
\dot{\mathcal{H}}_{i}= & -\left|\boldsymbol{v}_{i}\right|^{2}-\left|\boldsymbol{\Phi}^{\top}\left(\boldsymbol{\xi}_{i}\right) \mathbf{e}_{i}\right|^{2}-\left|\mathbf{Y}_{K i} \tilde{\boldsymbol{\theta}}_{K i}\right|^{2}-2 \sum_{j \in \mathcal{N}_{i}} w_{i j} \dot{\mathbf{x}}_{i}^{\top}\left(\mathbf{x}_{i}-\mathbf{x}_{j}\left(t-T_{j i}(t)\right)\right)+ \\
& +\sum_{j \in \mathcal{N}_{i}} w_{i j}\left(\mathbf{x}_{i}-\mathbf{x}_{j}\right)^{\top}\left(\dot{\mathbf{x}}_{i}-\dot{\mathbf{x}}_{j}\right) .
\end{aligned}
$$


From the fact that $\int_{t-T_{j i}(t)}^{t} \dot{\mathbf{x}}_{j}(\sigma) d \sigma=\mathbf{x}_{j}-\mathbf{x}_{j}\left(t-T_{j i}(t)\right)$ and doing some simplifications, finally yields

$$
\dot{\mathcal{H}}_{i}=-\left|\boldsymbol{v}_{i}\right|^{2}-\left|\boldsymbol{\Phi}^{\top}\left(\boldsymbol{\xi}_{i}\right) \mathbf{e}_{i}\right|^{2}-\left|\mathbf{Y}_{K i} \tilde{\boldsymbol{\theta}}_{K i}\right|^{2}-\sum_{j \in \mathcal{N}_{i}} w_{i j}\left(2 \dot{\mathbf{x}}_{i}^{\top} \int_{t-T_{j i}(t)}^{t} \dot{\mathbf{x}}_{j}(\sigma) d \sigma+\left(\dot{\mathbf{x}}_{i}+\dot{\mathbf{x}}_{j}\right)^{\top}\left(\mathbf{x}_{i}-\mathbf{x}_{j}\right)\right)
$$

The last term $\left(\dot{\mathbf{x}}_{i}+\dot{\mathbf{x}}_{j}\right)^{\top}\left(\mathbf{x}_{i}-\mathbf{x}_{j}\right)$ can be expressed as

$$
\left(\dot{\mathbf{x}}_{i}+\dot{\mathbf{x}}_{j}\right)^{\top}\left(\mathbf{x}_{i}-\mathbf{x}_{j}\right)=-\mathbf{x}_{i}^{\top}\left(\dot{\mathbf{x}}_{i}-\dot{\mathbf{x}}_{j}\right)+\dot{\mathbf{x}}_{i}^{\top}\left(\mathbf{x}_{i}-\mathbf{x}_{j}\right)-\mathbf{x}_{j}^{\top} \dot{\mathbf{x}}_{j}+\mathbf{x}_{i}^{\top} \dot{\mathbf{x}}_{i}
$$

defining $\mathbf{Q}:=\left[\mathbf{x}_{1}^{\top} \dot{\mathbf{x}}_{1}, \ldots, \mathbf{x}_{N}^{\top} \dot{\mathbf{x}}_{N}\right]^{\top}$, using (3) and because $\sum_{i \in \bar{N}} \sum_{j \in \mathcal{N}_{i}} w_{i j}\left(Q_{i}-Q_{j}\right)=\mathbf{1}_{N}^{\top} \mathbf{L Q}=0$, it is straightforward to show that

$$
\sum_{i \in \bar{N}} \sum_{j \in \mathcal{N}_{i}} w_{i j}\left(\dot{\mathbf{x}}_{i}+\dot{\mathbf{x}}_{j}\right)^{\top}\left(\mathbf{x}_{i}-\mathbf{x}_{j}\right)=\mathbf{x}^{\top}\left(\left(\mathbf{L}-\mathbf{L}^{\top}\right) \otimes \mathbf{I}_{7}\right) \dot{\mathbf{x}}=0
$$

where $\mathbf{x}:=\left[\mathbf{x}_{1}^{\top}, \ldots, \mathbf{x}_{N}^{\top}\right]^{\top}, \dot{\mathbf{x}}:=\left[\dot{\mathbf{x}}_{1}^{\top}, \ldots, \dot{\mathbf{x}}_{N}^{\top}\right]^{\top} \in \mathbb{R}^{7 N}$. Hence, taking $\mathcal{H}=\sum_{i \in \bar{N}} \mathcal{H}_{i}$ and invoking P6, it holds that

$$
\dot{\mathcal{H}}=-\sum_{i \in \bar{N}}\left[\left|\dot{\mathbf{x}}_{i}\right|^{2}+3\left|\dot{\boldsymbol{\xi}}_{i}\right|^{2}+\left|\boldsymbol{\Phi}^{\top}\left(\boldsymbol{\xi}_{i}\right) \mathbf{e}_{i}\right|^{2}+\left|\mathbf{Y}_{K i} \tilde{\boldsymbol{\theta}}_{K i}\right|^{2}+2 \sum_{j \in \mathcal{N}_{i}} w_{i j} \dot{\mathbf{x}}_{i}^{\top} \int_{t-T_{j i}(t)}^{t} \dot{\mathbf{x}}_{j}(\sigma) d \sigma\right]
$$

$\mathcal{H}$ does not qualify as a Lyapunov Function, that is, it does not satisfy $\dot{\mathcal{H}}<0$. And then, in the same spirit as in [31] and in order to get the below inequality, we integrate $\dot{\mathcal{H}}$ from 0 to $t$ and apply Lemma 1 of [55] to the double integral terms with $\alpha_{i} \in \mathbb{R}_{>0}$. This yields

$\mathcal{H}(0) \geqslant \mathcal{H}(t)+\sum_{i \in \bar{N}}\left(\left\|\boldsymbol{\Phi}^{\top}\left(\boldsymbol{\xi}_{i}\right) \mathbf{e}_{i}\right\|_{2}^{2}+\left\|\mathbf{Y}_{K i} \tilde{\boldsymbol{\theta}}_{K i}\right\|_{2}^{2}+\sum_{j \in \mathcal{N}_{i}} w_{i j}\left[\left(\frac{1}{\ell_{i i}}-\alpha_{i}\right)\left\|\dot{\mathbf{x}}_{i}\right\|_{2}^{2}-\frac{{ }^{*} T_{j i}^{2}}{\alpha_{i}}\left\|\dot{\mathbf{x}}_{j}\right\|_{2}^{2}\right]\right)$,

where the fact that $\ell_{i i}:=\sum_{j \in \mathcal{N}_{i}} w_{i j}$ has also been used. Defining the matrix $\Psi \in \mathbb{R}^{N \times N}$ as

$$
\boldsymbol{\Psi}:=\left[\begin{array}{cccc}
1-\ell_{11} \alpha_{1} & -\frac{w_{12} T_{21}^{2}}{\alpha_{1}} & \cdots & -\frac{w_{1 N} T_{N 1}^{2}}{\alpha_{1}} \\
-\frac{w_{12} T_{12}^{2}}{\alpha_{2}} & 1-\ell_{22} \alpha_{2} & \cdots & -\frac{w_{2 N} T_{N 2}^{2}}{\alpha_{2}} \\
\vdots & \vdots & \ddots & \vdots \\
-\frac{w_{1 N} T_{1 N}^{2}}{\alpha_{N}} & -\frac{w_{2 N} T_{2 N}^{2}}{\alpha_{N}} & \cdots & 1-\ell_{N N} \alpha_{N}
\end{array}\right],
$$

yields $\mathcal{H}(0) \geqslant \mathcal{H}(t)+\sum_{i \in \bar{N}}\left(\left\|\boldsymbol{\Phi}^{\top}\left(\boldsymbol{\xi}_{i}\right) \mathbf{e}_{i}\right\|_{2}^{2}+\left\|\mathbf{Y}_{K i} \tilde{\boldsymbol{\theta}}_{K i}\right\|_{2}^{2}\right)+\mathbf{1}_{N}^{\top} \boldsymbol{\Psi}\left[\left\|\dot{\mathbf{x}}_{1}\right\|_{2}^{2}, \ldots,\left\|\dot{\mathbf{x}}_{N}\right\|_{2}^{2}\right]^{\top}$.

Setting all $\alpha_{i}$ and the interconnection weights $w_{i j}$ such that

$$
\left.1>\sum_{j \in \mathcal{N}_{i}} w_{i j} \quad \alpha_{i}+\frac{{ }^{*} T_{i j}^{2}}{\alpha_{j}}\right)
$$

then there exists $\boldsymbol{\mu} \in \mathbb{R}^{N}$, defined as $\boldsymbol{\mu}:=\boldsymbol{\Psi}^{\top} \mathbf{1}_{N}$, such that $\mu_{i}>0$, for all $i \in \bar{N}$. Hence, 


$$
\mathcal{H}(0) \geqslant \mathcal{H}(t)+\sum_{i \in \bar{N}}\left(\left\|\boldsymbol{\Phi}^{\top}\left(\boldsymbol{\xi}_{i}\right) \mathbf{e}_{i}\right\|_{2}^{2}+\left\|\mathbf{Y}_{K i} \tilde{\boldsymbol{\theta}}_{K i}\right\|_{2}^{2}+\mu_{i}\left\|\dot{\mathbf{x}}_{i}\right\|_{2}^{2}\right)
$$

Because $\mathcal{H}(0)$ is a positive constant and $\mathcal{H}(t)>0$, then $\boldsymbol{\Phi}^{\top}\left(\boldsymbol{\xi}_{i}\right) \mathbf{e}_{i}, \mathbf{Y}_{K i} \tilde{\boldsymbol{\theta}}_{K i}, \dot{\mathbf{x}}_{i} \in \mathcal{L}_{2}$ and $\mathcal{H} \in$ $\mathcal{L}_{\infty}$. Furthermore, $\mathcal{H}$ is positive definite and radially unbounded with regards to $\boldsymbol{\epsilon}_{i}, \tilde{\boldsymbol{\theta}}_{D i}, \tilde{\boldsymbol{\theta}}_{K i}, \mid \mathbf{x}_{i}-$ $\mathbf{x}_{\ell}|,| \mathbf{x}_{i}-\mathbf{x}_{j} \mid$, hence, $\mathcal{H} \in \mathcal{L}_{\infty}$ ensures that all these signals are also bounded.

On one hand, P8, $\dot{\mathbf{x}}_{i} \in \mathcal{L}_{2}$ and $\tilde{\boldsymbol{\theta}}_{K i},\left|\mathbf{x}_{i}-\mathbf{x}_{\ell}\right|,\left|\mathbf{x}_{i}-\mathbf{x}_{j}\right| \in \mathcal{L}_{\infty}$, for all $i \in \bar{N}$ and $j \in \mathcal{N}_{i}$, together with $\mathbf{P 4}$ imply that $\boldsymbol{\Phi}^{\top}\left(\boldsymbol{\xi}_{i}\right) \mathbf{e}_{i}, \boldsymbol{\phi}_{i} \in \mathcal{L}_{\infty}$. On the other hand, $\boldsymbol{\epsilon}_{i}, \boldsymbol{\phi}_{i} \in \mathcal{L}_{\infty}$ ensures that $\dot{\mathbf{q}}_{i} \in \mathcal{L}_{\infty}$, which together with $\mathbf{P 4}$ and P8, implies that $\dot{\mathbf{x}}_{i} \in \mathcal{L}_{\infty}$.

Using (22), it can be shown that $\dot{\mathbf{e}}_{i}=a_{i} \dot{\mathbf{x}}_{i}+\sum_{j \in \mathcal{N}_{i}} w_{i j}\left[\dot{\mathbf{x}}_{i}-\left(1-\dot{T}_{j i}(t)\right) \dot{\mathbf{x}}_{j}\left(t-T_{j i}(t)\right)\right]$, and boundedness of $\dot{\mathbf{x}}_{i}$ and $\mathbf{A 2}$ support the fact that $\dot{\mathbf{e}}_{i}$ is also bounded. Furthermore, $\dot{\boldsymbol{\phi}}_{i}$ satisfies

$$
\begin{aligned}
\dot{\boldsymbol{\phi}}_{i} & =\dot{\hat{\mathbf{J}}}_{i}^{\dagger}\left(\mathbf{q}_{i}\right) \boldsymbol{\Phi}^{\top}\left(\boldsymbol{\xi}_{i}\right) \mathbf{e}_{i}+\hat{\mathbf{J}}_{i}^{\dagger}\left(\mathbf{q}_{i}\right) \frac{d}{d t}\left(\boldsymbol{\Phi}^{\top}\left(\xi_{i}\right) \mathbf{e}_{i}\right) \\
& =\dot{\hat{\mathbf{J}}}_{i}^{\dagger}\left(\mathbf{q}_{i}\right) \boldsymbol{\Phi}^{\top}\left(\xi_{i}\right) \mathbf{e}_{i}+\hat{\mathbf{J}}_{i}^{\dagger}\left(\mathbf{q}_{i}\right)\left[\dot{\boldsymbol{\Phi}}^{\top}\left(\xi_{i}\right) \mathbf{e}_{i}+\boldsymbol{\Phi}^{\top}\left(\xi_{i}\right) \dot{\mathbf{e}}_{i}\right] .
\end{aligned}
$$

Hence assumption A2, properties $\mathbf{P 4}$ and $\mathbf{P 8}$ and boundedness of $\dot{\mathbf{q}}_{i}, \dot{\mathbf{x}}_{i}$ and $\dot{\mathbf{e}}_{i}$ imply that $\dot{\boldsymbol{\phi}}_{i} \in \mathcal{L}_{\infty}$ and, consequently, $\frac{d}{d t}\left(\boldsymbol{\Phi}^{\top}\left(\boldsymbol{\xi}_{i}\right) \mathbf{e}_{i}\right) \in \mathcal{L}_{\infty}$.

Now, boundedness of all these signals ensure, from the closed-loop system (15), that $\dot{\boldsymbol{\epsilon}}_{i} \in$ $\mathcal{L}_{\infty}$. Because $\dot{\boldsymbol{\epsilon}}_{i}=\ddot{\mathbf{q}}_{i}+\dot{\boldsymbol{\phi}}_{i}, \ddot{\mathbf{q}}_{i} \in \mathcal{L}_{\infty}$. Furthermore, $\ddot{\mathbf{x}}_{i}=\boldsymbol{\Phi}^{\top}\left(\xi_{i}\right)\left[\mathbf{J}_{i}\left(\mathbf{q}_{i}\right) \ddot{\mathbf{q}}_{i}+\dot{\mathbf{J}}_{i}\left(\mathbf{q}_{i}\right) \dot{\mathbf{q}}_{i}\right]+$ $\dot{\boldsymbol{\Phi}}^{\top}\left(\boldsymbol{\xi}_{i}\right) \mathbf{J}_{i}\left(\mathbf{q}_{i}\right) \dot{\mathbf{q}}_{i}$, thus $\mathbf{P 4}, \mathbf{P 8}$ and $\dot{\mathbf{x}}_{i}, \dot{\mathbf{q}}_{i}, \ddot{\mathbf{q}}_{i} \in \mathcal{L}_{\infty}$ ensure that $\ddot{\mathbf{x}}_{i} \in \mathcal{L}_{\infty}$.

Finally, $\boldsymbol{\Phi}^{\top}\left(\boldsymbol{\xi}_{i}\right) \mathbf{e}_{i}, \dot{\mathbf{x}}_{i} \in \mathcal{L}_{2} \cap \mathcal{L}_{\infty}$ and $\frac{d}{d t}\left(\boldsymbol{\Phi}^{\top}\left(\xi_{i}\right) \mathbf{e}_{i}\right), \ddot{\mathbf{x}}_{i} \in \mathcal{L}_{\infty}$ yields, by Barbălat's Lemma, $\lim _{t \rightarrow \infty}\left|\boldsymbol{\Phi}^{\top}\left(\boldsymbol{\xi}_{i}(t)\right) \mathbf{e}_{i}(t)\right|=0, \lim _{t \rightarrow \infty}\left|\dot{\mathbf{x}}_{i}(t)\right|=0$ and, from P6, $\lim _{t \rightarrow \infty}\left|\boldsymbol{v}_{i}(t)\right|=0$.

Before presenting one of the main results, it should be mentioned that, although $\xi_{i}=\xi_{\ell}$ and $\xi_{i}=-\xi_{\ell}$ represent the same physical orientation, the closed-loop system (15) has two possible equilibria. However, $\boldsymbol{\xi}_{i}=-\boldsymbol{\xi}_{\ell}$ corresponds to an unstable equilibrium point.

\section{Remark 2}

In order to obtain an interpretation of the stability condition (26), first set, for all $i \in \bar{N}, \alpha_{i}=\alpha$ for any $\alpha>0$. In this case (21) transforms to $1>\sum_{j \in \mathcal{N}_{i}} w_{i j}\left(\alpha+\frac{{ }^{*} T_{i j}^{2}}{\alpha}\right)$. Doing some algebra and noting that $\ell_{i i}:=\sum_{j \in \mathcal{N}_{i}} w_{i j}$, we obtain the inequality $\ell_{i i} \alpha^{2}-\alpha+\sum_{j \in \mathcal{N}_{i}} w_{i j}{ }^{*} T_{i j}^{2}<0$.

Clearly, such inequality is satisfied if there exist real solutions for the second order equation $\ell_{i i} \alpha^{2}-\alpha+\sum_{j \in \mathcal{N}_{i}} w_{i j}{ }^{*} T_{i j}^{2}=0$. In fact, such solutions exist if and only if $1>4 \ell_{i i} \sum_{j \in \mathcal{N}_{i}} w_{i j}{ }^{*} T_{i j}^{2}$.

This last inequality is equivalent to (26). Because the bounds of the time-delays are given a priori, the only free parameters in this last inequality are the interconnection weights. Obviously, when delays are negligible any interconnection weights satisfy this inequality. However, for large delays the interconnection weights have to be small.

\section{Proposition 1}

Suppose that Assumptions A1, A2, and A3 hold. Additionally assume that, for any $\alpha_{i}>0$, condition (26) holds. And then, the controller given by (13), (14), (21), and (27) together with the dynamic and kinematic parameter adaptation laws (17) and (20), respectively, solves the LFCP everywhere except when $\left(\dot{\mathbf{x}}_{i}(0), \mathbf{p}_{i}(0), \xi_{i}(0)\right)=\left(\mathbf{0}_{7}, \mathbf{p}_{\ell},-\boldsymbol{\xi}_{\ell}\right)$ for all $i \in \bar{N}$.

\section{Proof}

First, note that $\lim _{t \rightarrow \infty}\left|\dot{\mathbf{x}}_{i}(t)\right|=0$ implies that $\int_{t-T_{j i}(t)}^{t} \dot{\mathbf{x}}_{j}(\sigma) d \sigma=\mathbf{x}_{j}-\mathbf{x}_{j}\left(t-T_{j i}(t)\right)=\mathbf{0}_{7}$. This and $\boldsymbol{\Phi}^{\top}\left(\xi_{i}\right) \mathbf{e}_{i}=\mathbf{0}_{6}$ ensure that 


$$
\begin{gathered}
a_{i}\left(\mathbf{p}_{i}-\mathbf{p}_{\ell}\right)+\sum_{j \in \mathcal{N}_{i}} w_{i j}\left(\mathbf{p}_{i}-\mathbf{p}_{j}\right)=\mathbf{0}_{3}, \\
a_{i} \mathbf{U}^{\top}\left(\boldsymbol{\xi}_{i}\right)\left(\boldsymbol{\xi}_{i}-\boldsymbol{\xi}_{\ell}\right)+\sum_{j \in \mathcal{N}_{i}} w_{i j} \mathbf{U}^{\top}\left(\boldsymbol{\xi}_{i}\right)\left(\boldsymbol{\xi}_{i}-\boldsymbol{\xi}_{j}\right)=\mathbf{0}_{3} .
\end{gathered}
$$

On one hand, defining $\mathbf{p}:=\left[\mathbf{p}_{1}^{\top}, \ldots, \mathbf{p}_{N}^{\top}\right]^{\top}$ allows to rewrite $(28)$ as $\left(\mathbf{A} \otimes \mathbf{I}_{3}\right)\left(\mathbf{p}-\left(\mathbf{1}_{N} \otimes \mathbf{p}_{\ell}\right)\right)+$ $\left(\mathbf{L} \otimes \mathbf{I}_{3}\right) \mathbf{p}=\mathbf{0}_{3 N}$ and, with the fact that $\mathbf{L} \mathbf{1}_{N}=\mathbf{0}_{N}$, (28) can be further written as $\left(\mathbf{L}_{\ell} \otimes \mathbf{I}_{3}\right)(\mathbf{p}-$ $\left.\left(\mathbf{1}_{N} \otimes \mathbf{p}_{\ell}\right)\right)=\mathbf{0}_{3 N}$, where $\mathbf{A}$ and $\mathbf{L}_{\ell}$ are defined in Proposition 1. Further, Proposition 1 and the Kronecker product properties ensure that $\operatorname{rank}\left(\mathbf{L}_{\ell} \otimes \mathbf{I}_{3}\right)=3 N$. Thus, for all $i \in \bar{N}, \mathbf{p}_{i}=\mathbf{p}_{\ell}$ is the only solution to (28).

On the other hand, defining $\xi:=\left[\xi_{1}^{\top}, \ldots, \xi_{N}^{\top}\right]^{\top}$ and $\overline{\mathbf{U}}(\xi):=\operatorname{diag}\left(\mathbf{U}\left(\xi_{1}\right), \ldots, \mathbf{U}\left(\xi_{N}\right)\right) \in$ $\mathbb{R}^{4 N \times 3 N}$, (29) can be written as

$$
\overline{\mathbf{U}}^{\top}(\boldsymbol{\xi})\left(\mathbf{A} \otimes \mathbf{I}_{4}\right)\left(\boldsymbol{\xi}-\left(\mathbf{1}_{N} \otimes \boldsymbol{\xi}_{\ell}\right)\right)+\overline{\mathbf{U}}^{\top}(\boldsymbol{\xi})\left(\mathbf{L} \otimes \mathbf{I}_{4}\right)\left(\boldsymbol{\xi}-\left(\mathbf{1}_{N} \otimes \boldsymbol{\xi}_{\ell}\right)\right)=\mathbf{0}_{3 N}
$$

or, what is the same, $\overline{\mathbf{U}}^{\top}(\boldsymbol{\xi})\left(\mathbf{L}_{\ell} \otimes \mathbf{I}_{4}\right)\left(\boldsymbol{\xi}-\left(\mathbf{1}_{N} \otimes \boldsymbol{\xi}_{\ell}\right)\right)=\mathbf{0}_{3 N}$. Defining $\mathbf{y}:=\left(\mathbf{L}_{\ell} \otimes \mathbf{I}_{4}\right)\left(\boldsymbol{\xi}-\left(\mathbf{1}_{N} \otimes\right.\right.$ $\left.\boldsymbol{\xi}_{\ell}\right)$ ), yields $\overline{\mathbf{U}}^{\top}(\boldsymbol{\xi}) \mathbf{y}=\mathbf{0}_{3 N}$. This equation has only two possible solutions, namely $\mathbf{y}=\mathbf{0}_{4 N}$ or $\mathbf{y} \in \operatorname{ker}\left(\overline{\mathbf{U}}^{\top}(\xi)\right)$.

The first solution $\mathbf{y}=\mathbf{0}_{4 N}$, implies that $\boldsymbol{\xi}=\left(\mathbf{1}_{N} \otimes \boldsymbol{\xi}_{\ell}\right)$, because $\left(\mathbf{L}_{\ell} \otimes \mathbf{I}_{4}\right)$ is of full rank. Hence, this implies consensus at $\boldsymbol{\xi}_{i}=\boldsymbol{\xi}_{\ell}$.

From Property P6, the second solution $\mathbf{y} \in \operatorname{ker}\left(\overline{\mathbf{U}}^{\top}(\xi)\right)$, implies that $\mathbf{y}=\left(\boldsymbol{\Delta} \otimes \mathbf{I}_{4}\right) \xi$, for $\boldsymbol{\Delta}:=$ $\operatorname{diag}\left(\delta_{i}\right) \in \mathbb{R}^{N \times N}$. Now, because $\mathbf{L}_{\ell}$ is of full rank, its inverse always exists, therefore the second solution is of the form

$$
\xi=\left(\left(\mathbf{I}_{N}-\mathbf{L}_{\ell}^{-1} \boldsymbol{\Delta}\right)^{-1} \otimes \mathbf{I}_{4}\right)\left(\mathbf{1}_{N} \otimes \boldsymbol{\xi}_{\ell}\right) .
$$

This expression implies that $\boldsymbol{\xi}$ is a linear combination of the vector $\left(\mathbf{1}_{N} \otimes \boldsymbol{\xi}_{\ell}\right)$. Finally, the normality condition of the quaternions, yields the second solution to (29) as $\boldsymbol{\xi}_{i}= \pm \boldsymbol{\xi}_{\ell}$, for all $i \in \bar{N}$.

Using (24) it can be easily shown that $\xi_{i}=\xi_{\ell}$ corresponds to a minimum energy point and, because $\mathcal{H}(t)$ is a decreasing function, that is, $\mathcal{H}(0) \geqslant \mathcal{H}(t)$ for all $t \geqslant 0$, any perturbation in the other equilibrium point $\xi_{i}=-\xi_{\ell}$ will drive the system to $\xi_{i}=\xi_{\ell}$. Hence, $\left(\boldsymbol{v}_{i}, \mathbf{p}_{i}, \boldsymbol{\xi}_{i}\right)=\left(\mathbf{0}_{6}, \mathbf{p}_{\ell}, \boldsymbol{\xi}_{\ell}\right)$ is asymptotically stable everywhere except at the unstable equilibrium point $\left(\boldsymbol{v}_{i}, \mathbf{p}_{i}, \boldsymbol{\xi}_{i}\right)=\left(\mathbf{0}_{\mathbf{6}}, \mathbf{p}_{\ell},-\boldsymbol{\xi}_{\ell}\right)$. This concludes the proof.

\section{Remark 3}

With similar assumptions to [1, 30, 48], the controller used in Proposition 1 can also solve the leader-follower problem for a dynamic leader provided that the leader trajectory is available (without delays) to all the agents and that it is bounded up to its third derivative. In such a case, the pose error (22) becomes

$$
\mathbf{e}_{i}=-\dot{\mathbf{x}}_{\ell}+\tilde{\mathbf{x}}_{i}+\sum_{j \in \mathcal{N}_{i}} w_{i j}\left[\mathbf{x}_{i}-\mathbf{x}_{j}\left(t-T_{j i}(t)\right)\right]
$$

where $\tilde{\mathbf{x}}_{i}:=\mathbf{x}_{i}-\mathbf{x}_{\ell}$ and $a_{i}>0$, for all $i \in \bar{N}$.

\subsection{Leaderless consensus problem}

The LCP is solved using the same controller as in the LFCP with the only difference being the absence of the leader and thus $a_{i}=0$, for all $i \in \bar{N}$.

\section{Proposition 2}

Suppose that Assumptions $\mathbf{A 1}$ and $\mathbf{A 2}$ hold. Additionally assume that, for any $\alpha_{i}>0$, condition (26) holds. And then, in the absence of a leader, the controller given by (13), (14), and (21) together with the dynamic and kinematic parameter adaptation laws (17) and (20), respectively, solves the LCP everywhere except when $\left(\dot{\mathbf{x}}_{i}(0), \mathbf{p}_{i}(0), \boldsymbol{\xi}_{i}(0)\right)=\left(\mathbf{0}_{7}, \mathbf{p}_{c},-\boldsymbol{\xi}_{c}\right)$ for all $i \in \bar{N}$. 
Proof

The proof follows verbatim the proof of Proposition 1, and hence, only the main steps are given.

In this case, $a_{i}=0$ and the error equation (22) becomes $\mathbf{e}_{i}=\sum_{j \in \mathcal{N}_{i}} w_{i j}\left[\mathbf{x}_{i}-\mathbf{x}_{j}\left(t-T_{j i}(t)\right)\right]$. Furthermore, the total energy-like function (24) transforms to $\mathcal{H}_{i}=\mathcal{W}_{i}+\frac{1}{2} \sum_{j \in \mathcal{N}_{i}} w_{i j}\left|\mathbf{x}_{i}-\mathbf{x}_{j}\right|^{2}$. Taking $\mathcal{H}=\sum_{i \in \bar{N}} \mathcal{H}_{i}$, yields the same time-derivative as in (25). Following the same procedure as in Section 3.2, by Barbǎlat's Lemma, it is proved that $\lim _{t \rightarrow \infty}\left|\boldsymbol{\Phi}^{\top}\left(\boldsymbol{\xi}_{i}(t)\right) \mathbf{e}_{i}(t)\right|=0$ and $\lim _{t \rightarrow \infty}\left|\dot{\mathbf{x}}_{i}(t)\right|=0$. In this case, (28) and (29), respectively, become

$$
\begin{gathered}
\sum_{j \in \mathcal{N}_{i}} w_{i j}\left(\mathbf{p}_{i}-\mathbf{p}_{j}\right)=\mathbf{0}_{3}, \\
\sum_{j \in \mathcal{N}_{i}} w_{i j} \mathbf{U}^{\top}\left(\boldsymbol{\xi}_{i}\right)\left(\boldsymbol{\xi}_{i}-\boldsymbol{\xi}_{j}\right)=\mathbf{0}_{3} .
\end{gathered}
$$

On one hand, (30) can be written as $\left(\mathbf{L} \otimes \mathbf{I}_{3}\right) \mathbf{p}=\mathbf{0}_{3 N}$. Because $\operatorname{rank}\left(\mathbf{L} \otimes \mathbf{I}_{3}\right)=3(N-1)$, $\mathbf{p}=\left(\mathbf{1}_{N} \otimes \mathbf{p}_{c}\right)$ is the only solution to (30), for any $\mathbf{p}_{c_{-}} \in \mathbb{R}^{3}$.

On the other hand, in matrix form, (31) is equal to $\overline{\mathbf{U}}^{\top}(\boldsymbol{\xi})\left(\mathbf{L} \otimes \mathbf{I}_{4}\right) \xi=\mathbf{0}_{3 N}$. Because $\operatorname{rank}(\mathbf{L})=$ $N-1$, the only possible solution to the equation $\left(\mathbf{L} \otimes \mathbf{I}_{4}\right) \xi=\mathbf{0}_{3 N}$ is $\boldsymbol{\xi}=\left(\mathbf{1}_{N} \otimes \boldsymbol{\xi}_{c}\right)$, for any $\boldsymbol{\xi}_{c} \in S^{3}$.

Now, from the fact that $\mathbf{U}^{\top}\left(\boldsymbol{\xi}_{i}\right) \boldsymbol{\xi}_{i}=\mathbf{0}_{3}$, (31) can be written as $\sum_{j \in \mathcal{N}_{i}} w_{i j} \mathbf{U}^{\top}\left(\boldsymbol{\xi}_{i}\right) \boldsymbol{\xi}_{j}=\mathbf{0}_{3}$. Therefore, there exists other possible solutions to (31) of the form $\xi_{j}=\delta_{j} \xi_{i}$, for any $\delta_{j} \in \mathbb{R}$. This last and the normality condition imply that $\boldsymbol{\xi}_{i}= \pm \boldsymbol{\xi}_{c}$, for all $i \in \vec{N}$.

Furthermore, $\xi_{i}=\xi_{c}$ corresponds to a minimum energy point and because $\mathcal{H}(t)$ is a decreasing function, any perturbation in $\boldsymbol{\xi}_{i}=-\boldsymbol{\xi}_{c}$ will drive the system to $\boldsymbol{\xi}_{i}=\boldsymbol{\xi}_{c}$. Hence, $\left(\boldsymbol{v}_{i}, \mathbf{p}_{i}, \boldsymbol{\xi}_{i}\right)=\left(\mathbf{0}_{6}, \mathbf{p}_{c}, \boldsymbol{\xi}_{c}\right)$ is asymptotically stable everywhere except at the unstable equilibrium point $\left(\boldsymbol{v}_{i}, \mathbf{p}_{i}, \boldsymbol{\xi}_{i}\right)=\left(\mathbf{0}_{6}, \mathbf{p}_{c},-\boldsymbol{\xi}_{c}\right)$. This concludes the proof.

\section{Remark 4}

When the interconnection time-delays are negligible, the pose error (22) becomes $\mathbf{e}_{i}=a_{i}\left(\mathbf{x}_{i}-\right.$ $\left.\mathbf{x}_{\ell}\right)+\sum_{j \in \mathcal{N}_{i}} w_{i j}\left(\mathbf{x}_{i}-\mathbf{x}_{j}\right)$ and thus (25) yields

$$
\dot{\mathcal{H}}=-\sum_{i \in \bar{N}}\left[\left|\dot{\mathbf{x}}_{i}\right|^{2}+3\left|\dot{\boldsymbol{\xi}}_{i}\right|^{2}+\left|\boldsymbol{\Phi}^{\top}\left(\boldsymbol{\xi}_{i}\right) \mathbf{e}_{i}\right|^{2}+\left|\mathbf{Y}_{K i} \tilde{\boldsymbol{\theta}}_{K i}\right|^{2}\right] .
$$

In this case, the proof of the solution to both consensus problems can be easily established invoking LaSalle's Invariance Theorem.

\section{Remark 5}

Following the same idea as in [1, 69] and because the robot manipulators often are redundant, that is, $n_{i}>6$, the proposed controller can be designed to solve the consensus problems and, at the same time, to perform a subtask objective (e.g., mechanical limit avoidance or obstacle avoidance). For example, let us propose the subtask error as $\left.\mathbf{e}_{s i}:=\left[\mathbf{I}_{n_{i}}-\hat{\mathbf{J}}_{i}^{\dagger}\left(\mathbf{q}_{i}\right)\right) \hat{\mathbf{J}}_{i}\left(\mathbf{q}_{i}\right)\right]\left(\dot{\mathbf{q}}_{i}-\dot{\mathbf{q}}_{d i}\right)$, where $\dot{\mathbf{q}}_{d i} \in \mathbb{R}^{n_{i}}$ is the desired velocity of the subtask. As usual in trajectory tracking control, $\dot{\mathbf{q}}_{d i}$ is assumed bounded up to its third derivative.

Redesigning (21) as $\boldsymbol{\phi}_{i}=\hat{\mathbf{J}}_{i}^{\dagger}\left(\mathbf{q}_{i}\right) \boldsymbol{\Phi}^{\top}\left(\boldsymbol{\xi}_{i}\right) \mathbf{e}_{i}-\left[\mathbf{I}_{n_{i}}-\hat{\mathbf{J}}_{i}^{\dagger}\left(\mathbf{q}_{i}\right) \hat{\mathbf{J}}_{i}\left(\mathbf{q}_{i}\right)\right] \dot{\mathbf{q}}_{d i}$ it holds that $\hat{\mathbf{J}}_{i}\left(\mathbf{q}_{i}\right) \boldsymbol{\phi}_{i}=$ $\boldsymbol{\Phi}^{\top}\left(\boldsymbol{\xi}_{i}\right) \mathbf{e}_{i}$, where the property of the Moore-Penrose pseudo-inverse matrix $\hat{\mathbf{J}}_{i}\left(\mathbf{q}_{i}\right) \hat{\mathbf{J}}_{i}^{\dagger}\left(\mathbf{q}_{i}\right)=\mathbf{I}_{6}$ has been used to obtain this expression. Thus, the rest of the proof remains the same for each consensus problem with the additional fact that $\mathbf{e}_{s i}$ converges to zero by noting that, in this case, $\boldsymbol{\epsilon}_{i}=\dot{\mathbf{q}}_{i}+\hat{\mathbf{J}}_{i}^{\dagger}\left(\mathbf{q}_{i}\right) \boldsymbol{\Phi}^{\top}\left(\boldsymbol{\xi}_{i}\right) \mathbf{e}_{i}-\left[\mathbf{I}_{n_{i}}-\hat{\mathbf{J}}_{i}^{\dagger}\left(\mathbf{q}_{i}\right) \hat{\mathbf{J}}_{i}\left(\mathbf{q}_{i}\right)\right] \dot{\mathbf{q}}_{d i}$ 
Thus, pre-multiplying the previous equation by $\left[\mathbf{I}_{n_{i}}-\hat{\mathbf{J}}_{i}^{\dagger}\left(\mathbf{q}_{i}\right) \hat{\mathbf{J}}_{i}\left(\mathbf{q}_{i}\right)\right]$ yields $\left[\mathbf{I}_{n_{i}}-\hat{\mathbf{J}}_{i}^{\dagger}\left(\mathbf{q}_{i}\right) \hat{\mathbf{J}}_{i}\left(\mathbf{q}_{i}\right)\right] \boldsymbol{\epsilon}_{i}=\mathbf{e}_{s i}$, hence, if $\boldsymbol{\epsilon}_{i}$ converges to zero so does $\mathbf{e}_{s i}$.

It should be underscored that the motions in the joint space because of $\dot{\mathbf{q}}_{d i}$ lie in the null space of $\hat{\mathbf{J}}_{i}\left(\mathbf{q}_{i}\right)$; thus, they do not induce any motion in the $S E(3)$.

\subsection{Human-robot interactions}

In the case that human torques, $\boldsymbol{\tau}_{h_{i}} \in \mathbb{R}^{n_{i}}$ are injected into one or multiple robots, the dynamic equation (1) changes to

$$
\mathbf{M}_{i}\left(\mathbf{q}_{i}\right) \ddot{\mathbf{q}}_{i}+\mathbf{C}_{i}\left(\mathbf{q}_{i}, \dot{\mathbf{q}}_{i}\right) \dot{\mathbf{q}}_{i}+\mathbf{g}_{i}\left(\mathbf{q}_{i}\right)=\boldsymbol{\tau}_{i}+c_{i} \boldsymbol{\tau}_{h_{i}},
$$

where $c_{i}=1$ if a human interacts with robot $i$ and $c_{i}=0$, otherwise.

When the human operator applies some forces, the equilibrium point is changed. However, because in the leader-follower case, the equilibrium point $\left(\boldsymbol{v}_{i}, \mathbf{p}_{i}, \boldsymbol{\xi}_{i}\right)=\left(\mathbf{0}_{6}, \mathbf{p}_{\ell}, \boldsymbol{\xi}_{\ell}\right)$ is almost globally attractive, once the human forces become zero, all robots converge asymptotically to the point $\left(\mathbf{0}_{6}, \mathbf{p}_{\ell}, \boldsymbol{\xi}_{\ell}\right)$.

In the leaderless case, the equilibrium point $\left(\boldsymbol{v}_{i}, \mathbf{p}_{i}, \boldsymbol{\xi}_{i}\right)=\left(\mathbf{0}_{6}, \mathbf{p}_{c}, \boldsymbol{\xi}_{c}\right)$ is almost globally attractive for some $\mathbf{p}_{c} \in \mathbb{R}^{3}$ and $\xi_{c} \in S^{3}$. When the human operator applies torques, to one or multiple robots, the consensus point is dynamically changed and when the human forces become zero, if the robots are not in consensus, they find another consensus point that might be different from the original point. This fact increases the applicability of the leaderless consensus algorithm in the sense that the robot network can be driven by one or multiple human operators, which allows a wide range of practical implementations such as formation control or teleoperation of networks of robots by one or multiple operators.

In this scenario, the following controller, derived from (13), is proposed

$$
\boldsymbol{\tau}_{i}=-\mathbf{Y}_{D i}\left(\mathbf{q}_{i}, \dot{\mathbf{q}}_{i}, \phi_{i}, \dot{\phi}_{i}\right) \hat{\boldsymbol{\theta}}_{D i}-K_{i} \boldsymbol{\epsilon}_{i}
$$

The closed-loop system (32) and (33) is

$$
\mathbf{M}_{i}\left(\mathbf{q}_{i}\right) \dot{\boldsymbol{\epsilon}}_{i}+\mathbf{C}_{i}\left(\mathbf{q}_{i}, \dot{\mathbf{q}}_{i}\right) \boldsymbol{\epsilon}_{i}+K_{i} \boldsymbol{\epsilon}_{i}=\mathbf{Y}_{D i} \tilde{\boldsymbol{\theta}}_{D i}+c_{i} \boldsymbol{\tau}_{h, i}
$$

\section{Proposition 3}

Consider the leaderless scenario and a robot network in which the kinematic and the dynamic parameters are uncertain and Assumptions A1 and A2 hold. Moreover, one or multiple human operators inject forces in one or multiple robot manipulators whose dynamics satisfy (32). And then, if the joint torques injected by the human are bounded, that is, $\boldsymbol{\tau}_{h, i} \in \mathcal{L}_{\infty}$, the controller given by (33), (14), and (21) together with the dynamic and kinematic parameter adaptation laws (17) and (20), ensures that $\epsilon_{i} \in \mathcal{L}_{\infty}$, for all $i \in \bar{N}$.

Proof

Using (16) and evaluating $\dot{\mathcal{V}}_{i}$ along (34) yields $\dot{\mathcal{V}}_{i}=-K_{i}\left|\boldsymbol{\epsilon}_{i}\right|^{2}+c_{i} \boldsymbol{\epsilon}_{i}^{\top} \boldsymbol{\tau}_{h, i}$. After applying Young's inequality, on the last term of the previous equation, it holds that

$$
\dot{\mathcal{V}}_{i} \leqslant-K_{i}\left|\boldsymbol{\epsilon}_{i}\right|^{2}+c_{i} \frac{K_{i}}{2}\left|\boldsymbol{\epsilon}_{i}\right|^{2}+c_{i} \frac{\left|\boldsymbol{\tau}_{h, i}\right|^{2}}{2 K_{i}}
$$

Note that if $c_{i}=0$, then $\dot{\mathcal{V}}_{i} \leqslant 0$ and boundedness of $\boldsymbol{\epsilon}_{i}$ follows directly. However, if $c_{i}=1$, then (35) can be simplified to

$$
\dot{\mathcal{V}}_{i} \leqslant-\frac{K_{i}}{2}\left|\epsilon_{i}\right|^{2}+\frac{\left|\tau_{h, i}\right|^{2}}{2 K_{i}}
$$


Because $\boldsymbol{\tau}_{h, i} \in \mathcal{L}_{\infty}$, then there exists $\gamma_{i}>0$ such that $\left|\boldsymbol{\tau}_{h, i}\right|^{2} \leqslant \gamma_{i}$. Therefore, if $\left|\boldsymbol{\epsilon}_{i}\right|^{2}>\frac{\gamma_{i}}{K_{i}^{2}}$ then $\dot{\mathcal{V}}_{i} \leqslant 0$. This last and the fact that $\mathcal{V}_{i}$ is positive definite and radially unbounded with regards to $\epsilon_{i}$ ensure that $\epsilon_{i} \in \mathcal{L}_{\infty}$. This concludes the proof.

\section{SIMULATIONS}

This section provides simulation results that show the effectiveness of the proposed consensus algorithms. Figure 1 depicts the simulated heterogeneous robot network, for the leader-follower and the leaderless consensus problems. It is composed of one 2-DoF (node 1), one 3-DoF (node 3) and two OMNI robots (Sensable, Wilmington, Massachusetts, United States), each with 3-DoF, (nodes 2 and 4). Table I presents the physical parameters and a description of these robots. The simulations have been carried out using Matlab's Simulink version 8.1. The direct kinematics of the robot manipulators are given by the following homogeneous matrices $\mathbf{H}(\mathbf{q}) \in \mathbb{R}^{4 \times 4}$.

$$
\begin{aligned}
\mathbf{H}_{2 D o F}(\mathbf{q}) & =\left[\begin{array}{cccc}
0 & -1 & 0 & 0 \\
s_{12} & 0 & c_{12} & l_{1} c_{1}+l_{2} c_{23} \\
-c_{12} & 0 & s_{12} & l_{1} c_{1}+l_{2} c_{23} \\
0 & 0 & 0 & 1
\end{array}\right] ; \\
\mathbf{H}_{3 D o F}(\mathbf{q}) & =\left[\begin{array}{cccc}
0 & -1 & 0 & 0 \\
s_{123} & 0 & c_{123} & l_{1} c_{1}+l_{2} c_{23}+l_{3} c_{123} \\
-c_{123} & 0 & s_{123} & l_{1} c_{1}+l_{2} c_{23}+l_{3} c_{123} \\
0 & 0 & 0 & 1
\end{array}\right] ; \\
\mathbf{H}_{O M N I}(\mathbf{q}) & =\left[\begin{array}{cccc}
-s_{1} s_{23} & -c_{1} & -s_{1} c_{23} & -s_{1}\left(l_{1} c_{2}+l_{2} c_{23}\right) \\
c_{1} s_{23} & -s_{1} & c_{1} c_{23} & c_{1}\left(l_{1} c_{2}+l_{2} c_{23}\right) \\
-c_{23} & 0 & s_{23} & l_{1} s_{2}+l_{2} s_{23} \\
0 & 0 & 0 & 1
\end{array}\right] ;
\end{aligned}
$$

where the notation $c_{12}, c_{123}, s_{12}$, and $s_{123}$ are abbreviations for $\cos \left(q_{1}+q_{2}\right), \cos \left(q_{1}+q_{2}+\right.$ $\left.q_{3}\right), \sin \left(q_{1}+q_{2}\right)$, and $\sin \left(q_{1}+q_{2}+q_{3}\right)$, respectively. The unit-quaternions have been derived from the rotation matrices following the algorithm proposed in [70]. The Jacobian matrices are given by

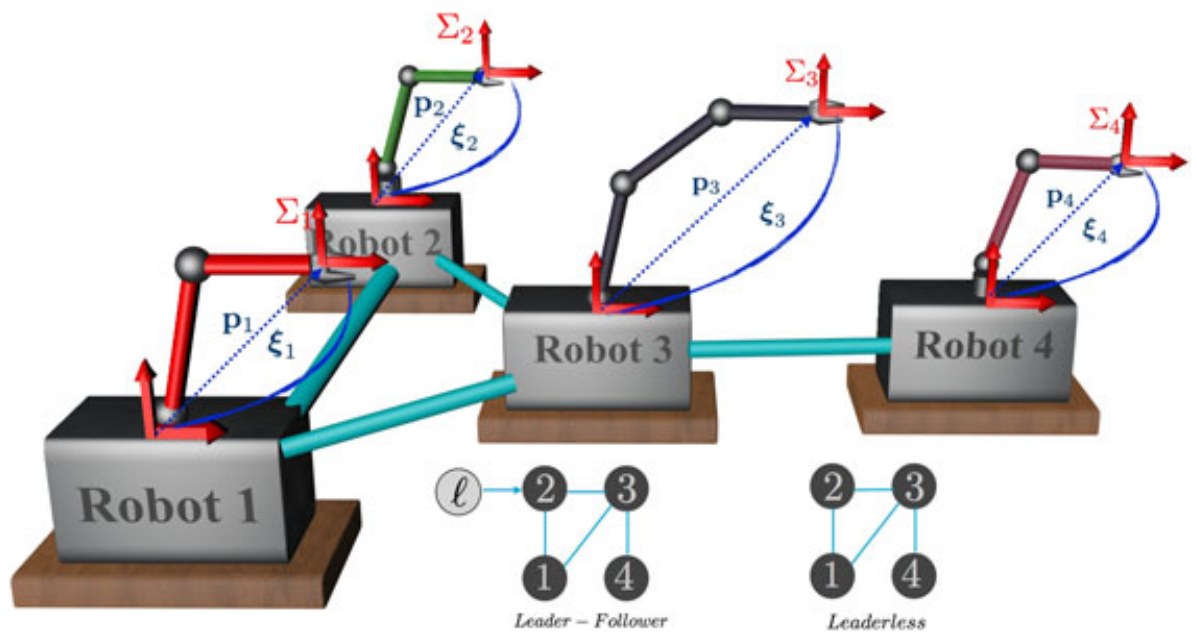

Figure 1. Simulated robot network and the two corresponding interconnection graphs. 


$$
\begin{aligned}
\mathbf{J}_{2 D o F}(\mathbf{q})= & {\left[\begin{array}{cc}
0 & 0 \\
-l_{2} s_{12}-l_{1} s_{1}-l_{3} s_{123} & -l_{2} s_{12}-l_{3} s_{123} \\
l_{2} c_{12}+l_{1} c_{1}+l_{3} c_{123} & l_{2} c_{12}+l_{3} c_{123} \\
1 & 1 \\
0 & 0 \\
0 & 0
\end{array}\right] ; } \\
\mathbf{J}_{3 D o F}(\mathbf{q})= & {\left[\begin{array}{ccc}
0 & 0 & 0 \\
-l_{2} s_{12}-l_{1} s_{1}-l_{3} s_{123} & -l_{2} s_{12}-l_{3} s_{123} & -l_{3} s_{123} \\
l_{2} c_{12}+l_{1} c_{1}+l_{3} c_{123} & l_{2} c_{12}+l_{3} c_{123} & l_{3} c_{123} \\
1 & 1 & 1 \\
0 & 0 & 0 \\
0 & 0 & 0
\end{array}\right] ; } \\
\mathbf{J}_{O M N I}(\mathbf{q})= & {\left[\begin{array}{ccc}
-s_{1}\left(l_{1} c_{2}+l_{2} c_{23}\right) & -c_{1}\left(l_{1} s_{2}+l_{2} s_{23}\right) & -l_{2} c_{1} s_{23} \\
c_{1}\left(l_{1} c_{2}+l_{2} c_{23}\right) & -s_{1}\left(l_{1} c_{2}+l_{2} c_{23}\right) & -l_{2} s_{1} s_{23} \\
0 & l_{2} c_{2}+l_{2} c_{23} & l_{2} c_{23} \\
0 & c_{1} & c_{1} \\
0 & s_{1} & s_{1} \\
1 & 0 & 0
\end{array}\right] ; }
\end{aligned}
$$

The kinematic regressors, $\mathbf{Y}_{K}(\mathbf{q}, \dot{\mathbf{q}})$ are as follows:

$$
\begin{aligned}
& \mathbf{Y}_{K, 2 D o F}=\left[\begin{array}{cc}
0 & 0 \\
-s_{1} \dot{q}_{1} & -s_{12} \dot{q}_{1}-s_{12} \dot{q}_{2} \\
c_{1} \dot{q}_{1} & c_{12} \dot{q}_{1}+c_{12} \dot{q}_{2} \\
0 & 0 \\
0 & 0 \\
0 & 0
\end{array}\right] \\
& \mathbf{Y}_{K, 3 D o F}=\left[\begin{array}{ccc}
0 & 0 & 0 \\
-s_{1} \dot{q}_{1} & -s_{12} \dot{q}_{1}-s_{12} \dot{q}_{2} & -s_{123} \dot{q}_{1}-s_{123} \dot{q}_{2}-s_{123} \dot{q}_{3} \\
c_{1} \dot{q}_{1} & c_{12} \dot{q}_{1}+c_{12} \dot{q}_{2} & c_{123} \dot{q}+c_{123} \dot{q}_{2}+c_{123} \dot{q}_{3} \\
0 & 0 & 0 \\
0 & 0 & 0 \\
0 & 0 & 0
\end{array}\right] \\
& \mathbf{Y}_{K, O M N I}=\left[\begin{array}{cc}
s_{1} s_{2} \dot{q}_{2}-c_{1} c_{2} \dot{q}_{1} & s_{23} s_{1} \dot{q}_{2}-c_{23} c_{1} \dot{q}_{1}+s_{23} s_{1} \dot{q}_{3} \\
-c_{2} c_{1} \dot{q}_{1}-c_{1} s_{2} \dot{q}_{2} & -c_{23} s_{1} \dot{q}_{1}-s_{23} c_{1} \dot{q}_{2}-s_{23} c_{1} \dot{q}_{3} \\
c_{2} \dot{q}_{2} & c_{23} \dot{q}_{2}+c_{23} \dot{q}_{3} \\
0 & 0 \\
0 & 0 \\
0 & 0
\end{array}\right]
\end{aligned}
$$

The kinematic parameter vectors are defined as $\boldsymbol{\theta}_{K, 2 D o F}=\left[l_{1}, l_{2}\right], \boldsymbol{\theta}_{K, 3 D o F}=\left[l_{1}, l_{2}, l_{3}\right]$ and $\boldsymbol{\theta}_{K, O M N I}=\left[l_{1}, l_{2}\right]$. The initial conditions for the estimated kinematic parameters are set to as follows: $\hat{\boldsymbol{\theta}}_{K, 2 \text { DoF }}(0)=[0.03,0.02]^{\top}, \hat{\boldsymbol{\theta}}_{K, 3 \mathrm{DoF}}(0)=[0.08,0.11,0.08]^{\top}, \hat{\boldsymbol{\theta}}_{K, \mathrm{OMNI}_{2}}(0)=$ $[0.05,0.01]^{\top}$, and $\hat{\boldsymbol{\theta}}_{K, O M N I_{4}}(0)=[0.04,0.02]^{\top}$, where $O M N I_{2}$ and $O M N I_{4}$ means OMNI of node 2 and OMNI of node 4 , respectively.

The nonlinear dynamic models of the robot manipulators are omitted for brevity but they can be found in $[1,46,71]$. The initial estimated dynamic parameters are set to zero. The variable timedelays between the nodes have been set to $T_{j i}=\rho+a_{1} \sin \left(\vartheta_{1} t\right)+a_{2} \sin \left(\vartheta_{2} t\right)$, and in Table II are listed the values of the parameters for all the interconnection's delays. 
Table I. Robots description and their physical parameters.

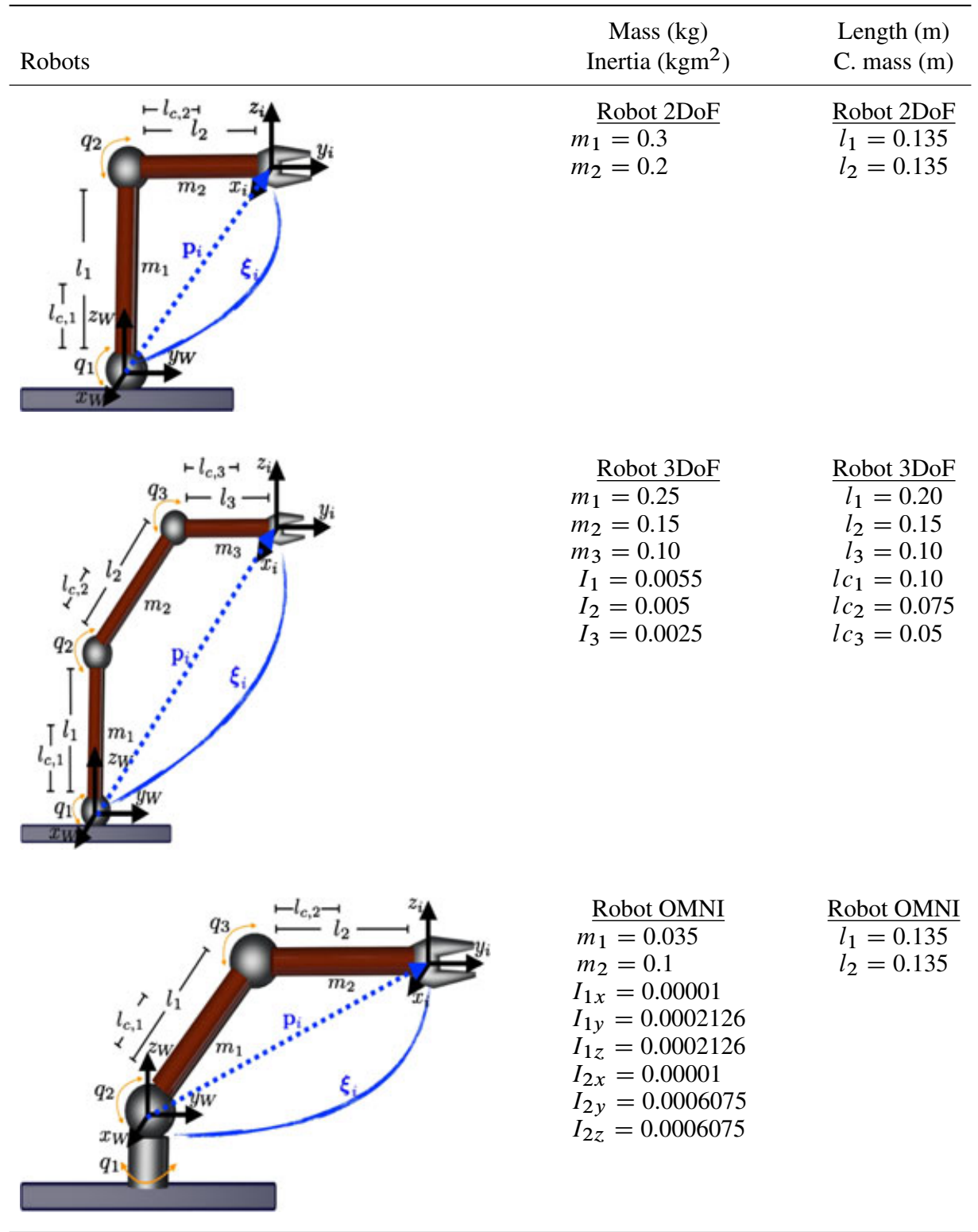

DoF, degrees-of-freedom.

Table II. Parameters of the variable time-delays for the expression $T_{j i}=\rho+a_{1} \sin \left(\vartheta_{1} t\right)+a_{2} \sin \left(\vartheta_{2} t\right)$.

\begin{tabular}{cccccc}
\hline Delay & $\rho$ & $a_{1}$ & $\vartheta_{1}(\mathrm{rad} / \mathrm{s})$ & $a_{2}$ & $\vartheta_{2}(\mathrm{rad} / \mathrm{s})$ \\
\hline$T_{21}$ & 0.12 & 0.05 & 7 & 0.06 & 27 \\
$T_{31}$ & 0.09 & 0.03 & 2 & 0.05 & 14 \\
$T_{12}$ & 0.14 & 0.03 & 5 & 0.08 & 23 \\
$T_{32}$ & 0.14 & 0.06 & 2 & 0.08 & 13 \\
$T_{13}$ & 0.11 & 0.04 & 3 & 0.07 & 27 \\
$T_{23}$ & 0.08 & 0.03 & 8 & 0.05 & 18 \\
$T_{43}$ & 0.13 & 0.05 & 4 & 0.07 & 29 \\
$T_{34}$ & 0.11 & 0.05 & 6 & 0.06 & 30 \\
\hline
\end{tabular}




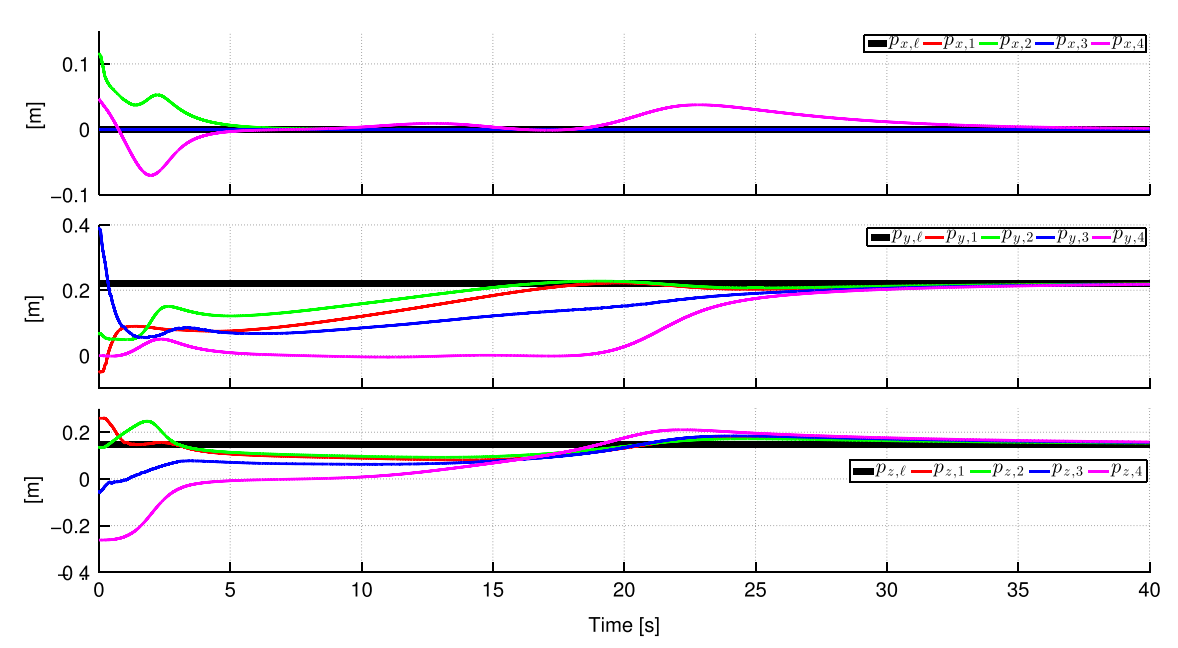

Figure 2. Robots position (leader-follower).

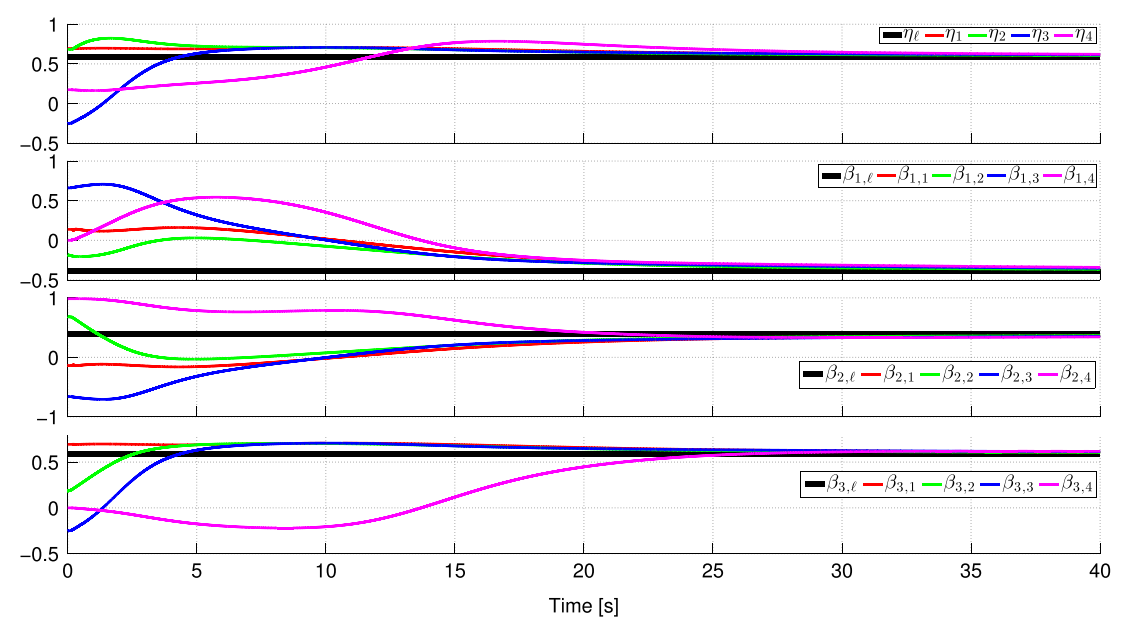

Figure 3. Robots orientation, represented by unit-quaternions (leader-follower).

\subsection{Leader-follower case}

The controller gains employed in this simulation are $K_{1}=30, K_{2}=25, K_{3}=30, K_{4}=50$, $\boldsymbol{\Gamma}_{D i}=80 \mathbf{I}_{9}$, and $\boldsymbol{\Gamma}_{K i}=50 \mathbf{I}_{4}$ for $i \in\{1,2,3,4\}$. For this case, only node 2 receives the leader constant pose $\mathbf{x}_{\ell}=[0,0.2202,0.1471,0.5879,-0.3928,0.3928,0.5879]^{\top}$, the interconnection weights $a_{i}$ are set to $a_{2}=1$ and $a_{i}=0$ for $i \in\{1,3,4\}$. The follower interconnection weights are the following: $w_{12}=w_{21}=1.5, w_{13}=w_{31}=0.8, w_{23}=w_{32}=0.7$, and $w_{34}=w_{43}=0.8$. These weights fulfill (26) using $\alpha_{1}=0.217, \alpha_{2}=0.259, \alpha_{3}=0.279$, and $\alpha_{4}=0.990$. Figures 2 and 3 show the position and the orientation dynamic behavior for the leader-follower control algorithm. It can be appreciated that the motion of the $2 \mathrm{DoF}$ and $3 \mathrm{DoF}$ robots only takes place in the plane defined by the coordinates $z$ and $y$, while that of the OMNI robots is in the 3D space and needs three coordinates. Furthermore, it is observed that despite the time-delays and the differences in the robots initial conditions, the robots asymptotically converge to the leader pose. Figure 4 depicts the kinematic parameter adaptation for each robot manipulator.

\subsection{Leaderless case}

The leaderless controller gains are $K_{1}=30, K_{2}=50, K_{3}=30, K_{4}=50, \Gamma_{D i}=80 \mathbf{I}_{9}$, and $\boldsymbol{\Gamma}_{K i}=50 \mathbf{I}_{4}$ for $i \in\{1,2,3,4\}$. In the leaderless consensus, $a_{i}=0$ for all $i \in\{1,2,3,4\}$. 


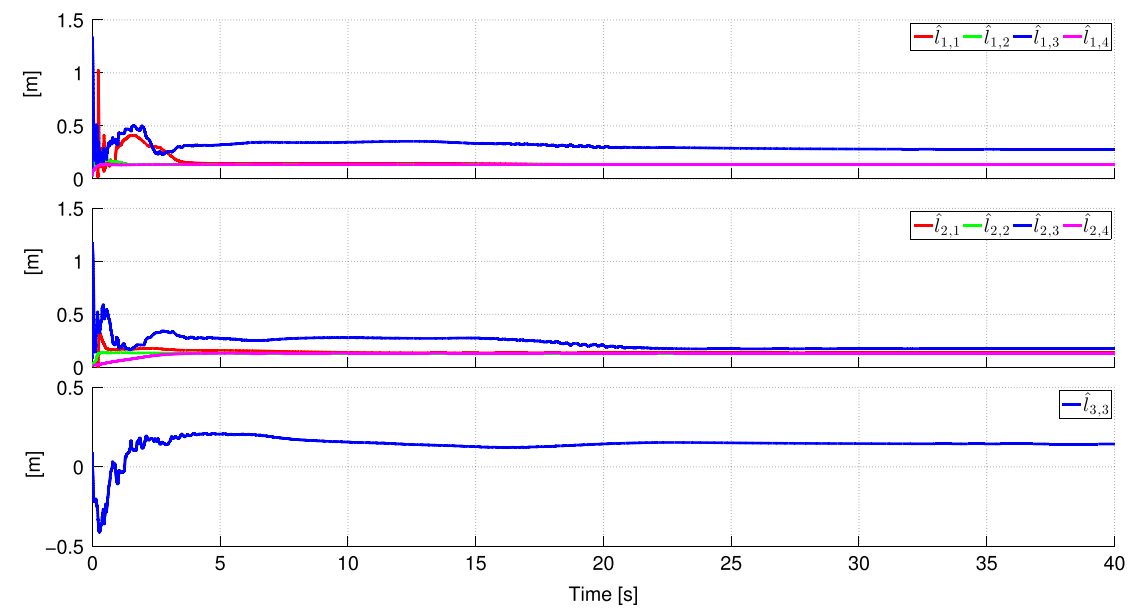

Figure 4. Kinematic parameters adaptation (leader-follower).

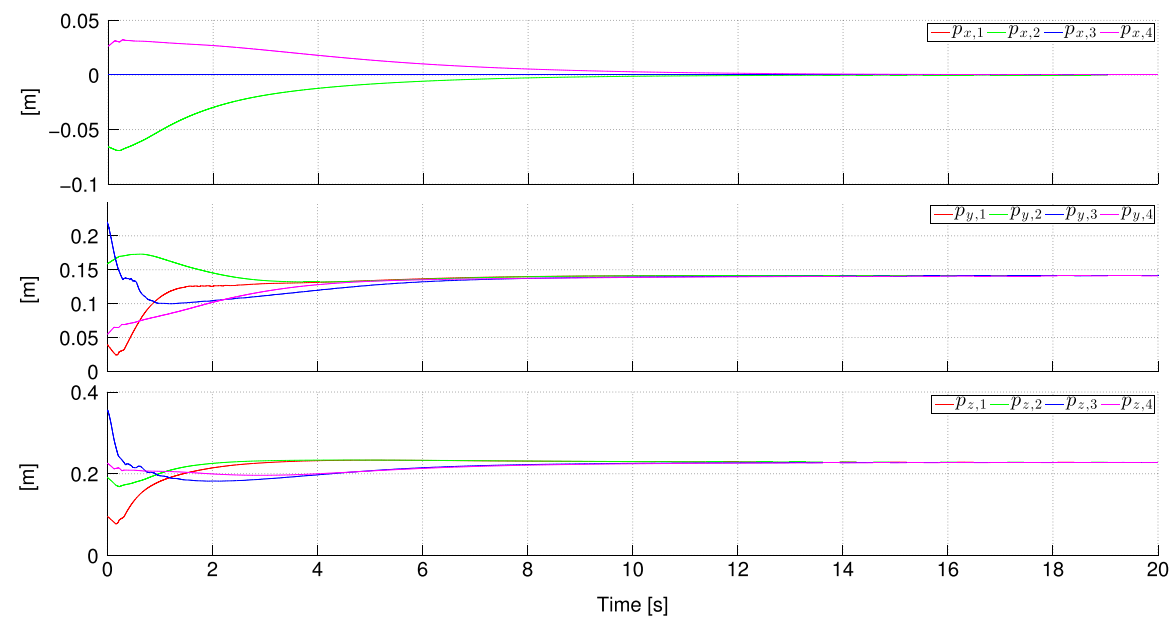

Figure 5. Robots position (leaderless).

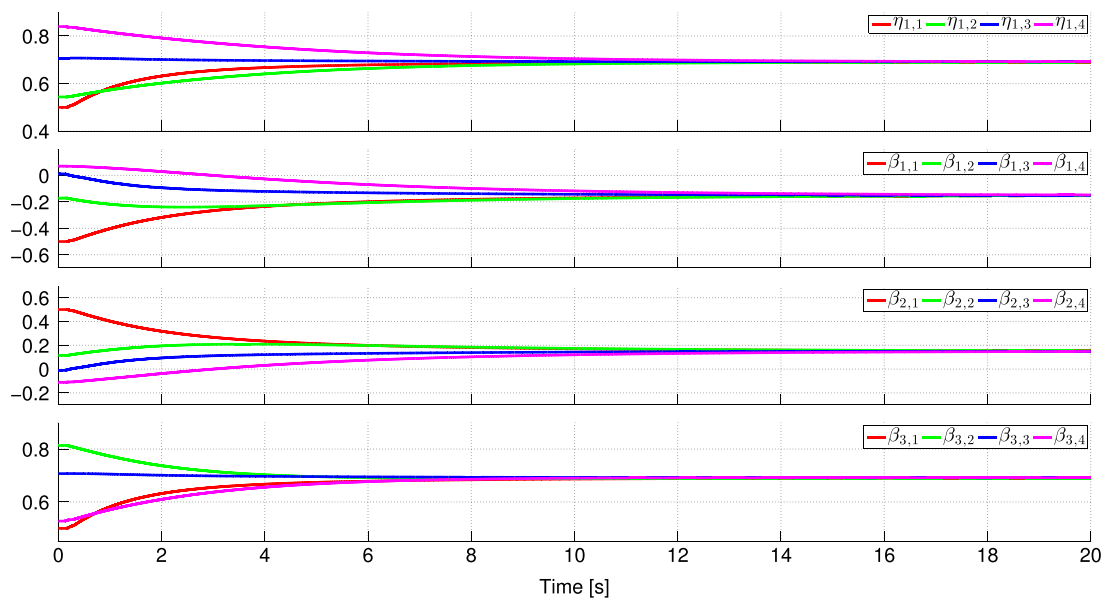

Figure 6. Robots orientation, represented by unit-quaternions (leaderless). 

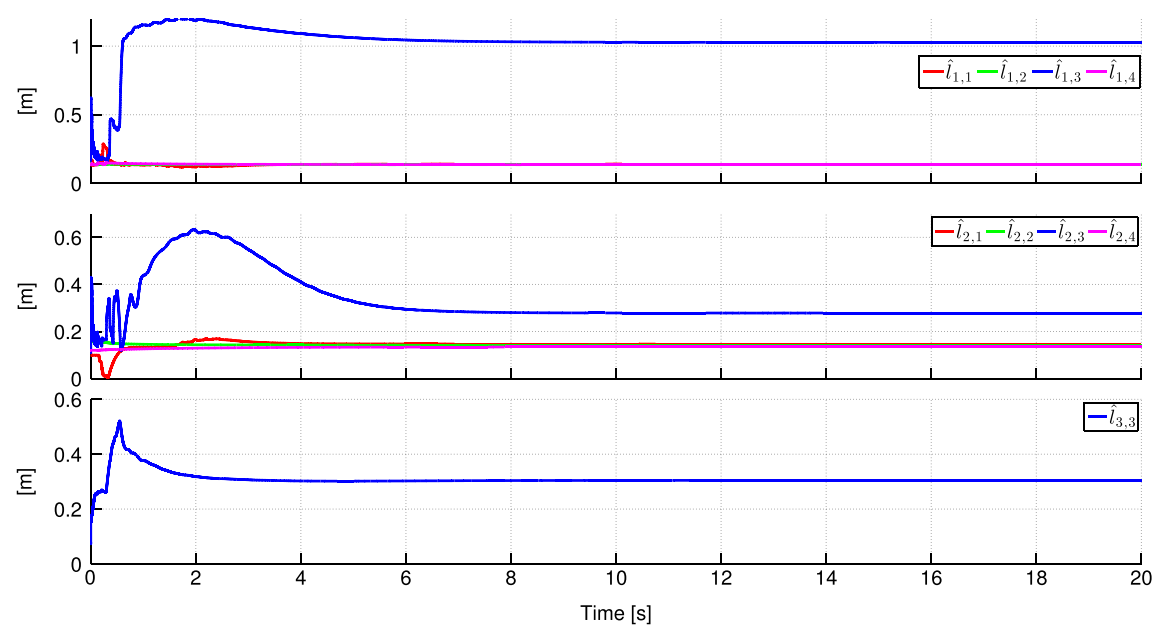

Figure 7. Kinematic parameters adaptation (leaderless).

The interconnection weights are $w_{12}=w_{21}=1, w_{13}=w_{31}=0.8, w_{23}=w_{32}=0.5$, and $w_{34}=w_{43}=1.2$. These weights satisfy condition (26) with $\alpha_{1}=0.30, \alpha_{2}=0.33, \alpha_{3}=0.25$, and $\alpha_{4}=0.43$. The robot network pose behavior is shown in Figure 5, for the position, and in Figure 6, for the orientation. It can be observed that the network asymptotically reaches a consensus pose. The kinematic estimated parameters are shown in Figure 7.

\section{CONCLUSIONS AND FUTURE WORK}

This paper proposes a novel adaptive controller that is capable of solving the leader-follower and the leaderless consensus problems in networks of heterogeneous robot manipulators in the task space. For the leader-follower case, it is only required that the leader pose be available to, at least, one follower. Moreover, the controller is robust to parameter uncertainty and to interconnection variable time-delays. Furthermore, the orientation of the robot end-effectors is represented by singularity-free unit-quaternions. A simple, yet useful, modification of the controller allows to deal with a dynamic leader and with additional subtask control objectives, like collisions and singularities avoidance. Simulations, using a network with four manipulators, are shown to illustrate the performance of the proposed scheme.

At present, we are working towards the solution of the leader-follower and the leaderless consensus problems for general directed graphs. Future research includes the extension of this work to the case when the interconnection graph is time-varying.

Similar to previous works, for example, [48, 49], the kinematic adaptation law (20) requires linear and angular velocity measurements. One possible solution to this issue is the incorporation of a velocity observer. This is another current research avenue.

\section{ACKNOWLEDGEMENTS}

This work has been partially supported by the Mexican CONACyT projects CB-129079 and INFR-229696 and by the Spanish MINECO projects DPI2011-22471, DPI2013-40882-P, DPI2014-57757-R and DPI2016-80077-R. The second author gratefully acknowledges the Mexican CONACyT for the Repatriación grant 262724.

\section{REFERENCES}

1. Liu Y, Chopra N. Controlled synchronization of heterogeneous robotic manipulators in the task space. IEEE Transactions on Robotics 2012; 28(1):268-275. 
2. Aldana C, Nuño E, Basañez L, Romero E. Operational space consensus of multiple heterogeneous robots without velocity measurements. Journal of the Franklin Institute 2014; 351(3):1517-1539.

3. Meng Z, Ren W, Cao Y, You Z. Leaderless and leader-following consensus with communication and input delays under a directed network topology. IEEE Transactions on Systems, Man, and Cybernetics, Part B 2011; 41(1):75-88.

4. Nuño E, Ortega R, Jayawardhana B, Basañez L. Coordination of multi-agent Euler-Lagrange systems via energyshaping: networking improves robustness. Automatica 2013; 49(10):3065-3071.

5. Liu Y, Min H, Wang S, Ma L, Liu Z. Consensus for multiple heterogeneous Euler-Lagrange systems with time-delay and jointly connected topologies. Journal of the Franklin Institute 2014; 351(6):3351-3363.

6. Rodriguez-Angeles A, Nijmeijer H. Mutual synchronization of robots via estimated state feedback: a cooperative approach. IEEE Transactions on Control Systems Technology 2004; 12(4):542-554.

7. Abdessameud A, Tayebi A, Polushin IG. Attitude synchronization of multiple rigid bodies with communication delays. IEEE Transactions on Automatic Control 2012; 57(9):2405-2411.

8. Hatanaka T, Igarashi Y, Fujita M, Spong M. Passivity-based pose synchronization in three dimensions. IEEE Transactions on Automatic Control 2012; 57(2):360-375.

9. Abdessameud A, Polushin IG, Tayebi A. Synchronization of nonlinear systems with communication delays and intermittent information exchange. Automatica 2015; 59:1-8.

10. Lee D, Spong M. Stable flocking of multiple inertial agents on balanced graphs. IEEE Transactions on Automatic Control 2007; 52(8):1469-1475.

11. Gu DB, Wang Z. Leader-follower flocking: algorithms and experiments. IEEE Transactions on Control Systems Technology 2009; 17(5):1211-1219.

12. Wang $\mathrm{X}$, Yu C, Lin Z. A dual quaternion solution to attitude and position control for rigid-body coordination. Transactions on Robotics 2012; 28(5):1162-1170.

13. Qin J, Zheng W, Gao H. Coordination of multiple agents with double-integrator dynamics under generalized interaction topologies. IEEE Transactions on Systems, Man, and Cybernetics, Part B 2012; 42(1):44-57.

14. Rutkovsky V, Somov S, Butyrin S, Sukhanov V. 19th ifac symposium on automatic control in aerospace attitude guidance and control of mini-satellites and free-flying robots*. IFAC Proceedings Volumes 2013; 46(19):399-404.

15. Maquin D, Furno L, Nielsen MC, Blanke M. 9th ifac symposium on fault detection, supervision and safety for technical processes, centralised versus decentralised control reconfiguration for collaborating underwater robots. IFAC-PapersOnLine 2015; 48(21):732-739.

16. Zhang K, Demetriou MA. Adaptation and optimization of the synchronization gains in the adaptive spacecraft attitude synchronization. Aerospace Science and Technology 2015; 46:116-123.

17. Nuño E, Basañez L, López-Franco C, Arana-Daniel N. Stability of nonlinear teleoperators using PD controllers without velocity measurements. Journal of the Franklin Institute 2014; 351(1):241-258.

18. Schwarzer F, Saha M, Latombe JC. Adaptive dynamic collision checking for single and multiple articulated robots in complex environments. IEEE Transactions on Robotics 2005; 21(3):338-353.

19. Gunn T, Anderson J. Dynamic heterogeneous team formation for robotic urban search and rescue. Journal of Computer and System Sciences 2015; 81(3):553-567. Special Issue on selected papers from the 4th International Conference on Ambient Systems, Networks and Technologies (ANT 2013).

20. Shukla A, Karki H. Application of robotics in onshore oil and gas industrya review part i. Robotics and Autonomous Systems 2016; 75, Part B:490-507.

21. Knepper RA, Layton T, Romanishin J, Rus D. Ikeabot: an autonomous multi-robot coordinated furniture assembly system. 2013 IEEE International Conference on Robotics and Automation (ICRA), Karlsruhe, 2013; 855-862.

22. Roa M A, Berenson D, Huang W. Mobile manipulation: toward smart manufacturing [tc spotlight]. IEEE Robotics Automation Magazine 2015; 22(4):14-15.

23. Bechar A, Vigneault C. Agricultural robots for field operations: concepts and components. Biosystems Engineering 2016; 149:94-111.

24. Olfati-Saber R, Murray R. Consensus problems in networks of agents with switching topology and time-delays. IEEE Transactions on Automatic Control 2004; 49(9):1520-1533.

25. Olfati-Saber R, Fax J, Murray R. Consensus and cooperation in networked multi-agent systems. Proceedings of the IEEE 2007; 95(1):215-233.

26. Ren W. On consensus algorithms for double-integrator dynamics. IEEE Transactions on Automatic Control 2008; 53(6):1503-1509.

27. Kim JM, Park JB, Choi YH. Leaderless and leader-following consensus for heterogeneous multi-agent systems with random link failures. IET Control Theory \& Applications 2014; 8(1):51-60.

28. Radenkovic M, Tadi M. Multi-agent adaptive consensus of networked systems on directed graphs. International Journal of Adaptive Control and Signal Processing 2016; 30(1):46-59. Acs.2577.

29. Dong W. On consensus algorithms of multiple uncertain mechanical systems with a reference trajectory. Automatica 2011; 47(9):2023-2028.

30. Nuño E, Ortega R, Basañez L, Hill D. Synchronization of networks of nonidentical Euler-Lagrange systems with uncertain parameters and communication delays. IEEE Transactions on Automatic Control 2011; 56(4):935-941.

31. Nuño E, Sarras I, Basañez L. Consensus in networks of nonidentical Euler-Lagrange systems using P+d controllers. IEEE Transactions on Robotics 2013; 26(6):1503-1508.

32. Ren W. Distributed leaderless consensus algorithms for networked Euler-Lagrange systems. International Journal of Control 2009; 82(11):2137-2149. 
33. Chung S, Slotine J. Cooperative robot control and concurrent synchronization of lagrangian systems. IEEE Transactions on Robotics 2009; 25(3):686-700.

34. Xu W, Cao J, Yu W, Lu J. Leader-following consensus of non-linear multi-agent systems with jointly connected topology. IET Control Theory \& Applications 2014; 8(6):432-440.

35. Meng Z, Dimarogonas D V, Johansson K. H. Leader-follower coordinated tracking of multiple heterogeneous lagrange systems using continuous control. IEEE Transactions on Robotics 2014; 30(3):739-745.

36. De Persis C, Jayawardhana B. Coordination of passive systems under quantized measurements. SIAM Journal on Control and Optimization 2012; 50(6):3155-3177.

37. Huang J, Wen C, Wang W, Song YD. Adaptive finite-time consensus control of a group of uncertain nonlinear mechanical systems. Automatica 2015; 51:292-301.

38. Wang B, Wang J, Zhang L, Zhang B. Robust adaptive consensus tracking for higher-order multi-agent uncertain systems with nonlinear dynamics via distributed intermittent communication protocol. International Journal of Adaptive Control and Signal Processing 2016; 30(3):511-533.

39. Wang F, Liu Z, Zhang Y, Chen B. Distributed adaptive coordination control for uncertain nonlinear multi-agent systems with dead-zone input. Journal of the Franklin Institute 2016; 353(10):2270-2289.

40. Mei J, Ren W, Ma G. Distributed coordinated tracking with a dynamic leader for multiple Euler-Lagrange systems. IEEE Transactions on Automatic Control 2011; 56(6):1415-1421.

41. Liu Y, Min H, Wang S, Liu Z, Liao S. Distributed consensus of a class of networked heterogeneous multi-agent systems. Journal of the Franklin Institute 2014; 351(3):1700-1716.

42. Ren W. Distributed attitude alignment in spacecraft formation flying. International Journal of Adaptive Control and Signal Processing 2007; 21(2-3):95-113.

43. Igarashi Y, Hatanaka T, Fujita M, Spong M. Passivity-based attitude synchronization in SE (3). IEEE Transactions on Control Systems Technology 2009; 17(5):1119-1134.

44. Weng S, Yue D, Yang T. Coordinated attitude motion control of multiple rigid bodies on manifold SO (3). IET Control Theory \& Applications 2013; 7(16):1984-1991.

45. Bai H, Arcak M, Wen J. Rigid body attitude coordination without inertial frame information. Automatica 2008; 44(12):3170-3175.

46. Aldana C, Nuño E, Basañez L. Bilateral teleoperation of cooperative manipulators. IEEE Int. Conf. Robot. Autom., Saint Paul, MN, 2012; 4274-4279.

47. Wang H, Xie Y. Task-space framework for bilateral teleoperation with time delays. Journal of Dynamic Systems, Measurement, and Control 2012; 134(5):51010-51020.

48. Wang H. Passivity based synchronization for networked robotic systems with uncertain kinematics and dynamics. Automatica 2013; 49(3):755-761.

49. Wang H. Task space synchronization of networked robotic systems with uncertain kinematics and dynamics. IEEE Transactions on Automatic Control 2013; 58(12):3169-3174.

50. Cheah C, Liu C, Slotine JJ. Adaptive jacobian tracking control of robots with uncertainties in kinematic, dynamic and actuator models. IEEE Transactions on Automatic Control 2006; 51(6):1024-1029.

51. Wang H, Xie Y. Adaptive inverse dynamics control of robots with uncertain kinematics and dynamics. Automatica 2009; 45(9):2114-2119.

52. Ghorbel F, Srinivasan B, Spong M. On the uniform boundedness of the inertia matrix of serial robot manipulators. Journal of Robotic Systems 1998; 15(1):17-28.

53. Kelly R, Santibáñez V, Loria A. Control of Robot Manipulators in Joint Space. Springer-Verlag: London, 2005.

54. Spong M, Hutchinson S, Vidyasagar M. Robot Modeling and Control. Wiley: Hoboken, NJ, 2005.

55. Nuño E, Basañez L, Ortega R, Spong M. Position tracking for nonlinear teleoperators with variable time-delay. International Journal of Robotics Research 2009; 28(7):895-910.

56. Arcak M. Passivity as a design tool for group coordination. IEEE Transactions on Automatic Control 2007; 52(8):1380-1390.

57. Sarlette A, Sepulchre R, Leonard N. Autonomous rigid body attitude synchronization. Automatica 2009; 45(2): $572-577$.

58. Nuño E, Ortega R, Jayawardhana B, Basañez L. Networking improves robustness in flexible-joint multi-robot systems with only joint position measurements. European Journal of Control 2013; 19(6):469-476.

59. Hong Y, Hu J, Gao L. Tracking control for multi-agent consensus with an active leader and variable topology. Automatica 2006; 42(7):1177-1182.

60. Cao Y, Ren W. Distributed Coordination of Multi-agent Networks: Emergent Problems, Models, and Issues. Springer-Verlag: London, 2011.

61. Lee D. Distributed backstepping control of multiple thrust-propelled vehicles on a balanced graph. Automatica 2012; 48(11):2971-2977.

62. Nuño E, Basañez L, Ortega R. Passivity-based control for bilateral teleoperation: a tutorial. Automatica 2011; 47(3):485-495.

63. Caccavale F, Siciliano B, Villani L. The role of Euler parameters in robot control. Asian Journal of Control 1999; 1(1):25-34.

64. Chou J, Quaternion kinematic and dynamic differential equations. IEEE Transactions on Robotics and Automation 1992; 8(1):53-64.

65. Kuipers JB. Quaternions and Rotation Sequences: A Primer with Applications to Orbits, Aerospace and Virtual Reality. Princeton University Press, 2002. 


\section{TASK SPACE CONSENSUS OF HETEROGENEOUS ROBOTIC SYSTEMS}

66. Wen JY, Kreutz-Delgado K. The attitude control problem. IEEE Transactions on Automatic Control 1991; 36(10): 1148-1162.

67. Fjellstad O. Control of unmanned underwater vehicles in six degrees of freedom: a quaternion feedback approach. PhD Thesis, Norwegian Institute of Technology, University of Trondheim, 1994.

68. Campa R, Camarillo K. Unit quaternions: a mathematical tool for modeling, path planning and control of robot manipulators. In Robot Manipulators, Ceccarelli M (ed.). InTech, 2008; 21-48.

69. Zergeroglu E, Walker DDI, Setlur P. Nonlinear tracking control of kinematically redundant robot manipulators. IEEE/ASME Transactions on Mechatronics 2004; 9(1):129-132.

70. Spurrier RA. Comment on singularity-free extraction of a quaternion from a direction-cosine matrix. Journal of Spacecraft and Rockets 1978; 15(4):255.

71. Nuño E, Ortega R, Basañez L. An adaptive controller for nonlinear bilateral teleoperators. Automatica 2010; 46(1):155-159. 